\title{
Cuadro de mando integral para la gestión del impacto social en organizaciones de empleo inclusivo
}

\section{Lina María Murillo Pérez}

RESUMEN: Los retos que presentan las organizaciones sociales en cuanto a la medición y visibilización del impacto social van en incremento. Las progresivas demandas de las comunidades, el mercado y la cooperación público-privada, exigen mayores esfuerzos en la búsqueda de eficacia y eficiencia de la acción social. Ante las múltiples debilidades en la planificación estratégica y los inexistentes o inadecuados sistemas de medición y control, que permitan conocer y gestionar los resultados de manera efectiva, se hace necesario proponer herramientas de gestión acopladas a las características, recursos y capacidades internas de estas organizaciones. El presente estudio busca desarrollar un instrumento para la gestión del impacto social de organizaciones 0 proyectos que tienen como misión fomentar el empleo inclusivo. La herramienta propuesta es una adaptación del Mapa Estratégico y el Cuadro de Mando Integral (CMI) a la empresa social. Como resultado del proyecto, se propone un CMI de 54 indicadores, cuantitativos y cualitativos, asociados a 21 objetivos estratégicos, en su mayoría, comunes a las organizaciones que trabajan por el empleo inclusivo. Dichos indicadores se definen a partir del análisis estratégico de dos organizaciones/proyectos que trabajan por la inclusión socio-laboral de colectivos vulnerables y, además, del análisis de diversas metodologías o enfoques ampliamente utilizados en la evaluación de desempeño social. Después de validar la herramienta, se concluye que su adopción puede resultar en diversos beneficios para las entidades sociales, no solo para la planificación y control de resultados, sino también para la tarea comunicativa y de rendición de cuentas.

PALABRAS CLAVE: Cuadro de mando integral, impacto social, empleo inclusivo, evaluación de proyectos, empresa social, balance social, desempeño social.

CLAVES ECONLIT: A13, L31, M14, O22.

Cómo citar este artículo / How to cite this article: MURILLO, L.M. (2020): "Cuadro de mando integral para la gestión del impacto social en organizaciones de empleo inclusivo", CIRIEC-España, Revista de Economía Pública, Social y Cooperativa, 98, 153-188. DOI: 107203/CIRIEC-E.98.13368.

Correspondencia: Lina María Murillo Pérez, Universidad Politécnica de Valencia, linamuri@gmail.com. 


\section{EXPANDED ABSTRACT}

\section{Balanced scorecard for the management of social impact in inclusive employment organizations}

The challenges presented by social organizations in terms of measurement and visibility of social impact are increasing. The progressive demands of the communities, the market and the public-private cooperation, require greater efforts to achieve efficacy and efficiency of social action. Given the multiple weaknesses in strategic planning and the nonexistent or inadequate measurement and control systems, that allow to know and to manage the results effectively, it is necessary to propose management tools tailored to the characteristics, resources and capabilities of these organizations.

According to these challenges, the main objective of the present study is to develop an instrument for managing the social impact of organizations or projects whose mission is to promote inclusive employment. This tool is based on an adaptation of the Strategic Map and the Balanced Scorecard (BSC), methodologies for strategic planning and management control, proposed by the authors Kaplan and Norton $(1996,2004)$.

These methodologies are considered extremely useful and relevant, not only because they have been validated in the conventional enterprise, but also because they are versatile, flexible and capable of being applied to any type of organization. The BSC, specifically, proposes financial and nonfinancial indicators in order to evaluate, through four perspectives (financial, customer, internal processes and learning and growth), the fulfillment of the value proposition of an organization in its different stakeholders. Thus, this instrument is also potentially applicable in the social organizations under study in order to manage and to control the fulfillment of their social objectives associated with various groups such as: beneficiaries, donors, government and employees, among others.

Additionally, this study establishes three specific objectives: a) To propose an adaptation of theoretical concepts related to the BSC according to the characteristics of the social enterprise, b) To define the pillars of strategic planning for social organizations or projects working for labor inclusion and c) To define a set of indicators (of results and impact) that, associated with the strategic objectives, serves as a guide for the measurement and management of social value.

Within this research, descriptive and practical, an analysis of the strategic planning of two social entities/projects, whose mission is to improve the labor insertion of groups at risk of exclusion, was carried out. These two organizations, specifically, develop their mission through training processes, labor intermediation, accompaniment or promotion of entrepreneurship. Taking into consideration this analysis and the review of other recognized approaches, methodologies and criteria for evaluating 
social performance, the first approximation of the tool object of study (strategic map and BSC) was developed.

The methodology used in this project included five stages, namely: a) the homologation of BSC concepts, b) the design of a Strategic Map, c) the analysis and definition of indicators, d) the development of the BSC and e) the validation and adjustment. In order to define the indicators that would conform the BSC, an analysis matrix was used, with which, the indicators from different models and standards of social evaluation and the indicators proposed by the organizations were compiled, contrasted and analyzed. Afterwards, through a process of weighing, valuing and prioritizing, the most relevant indicators were selected to be included into the tool.

It is necessary to clarify, that the validation stage consisted in the presentation of the initial model (Strategic Map and BSC) to two Valencian social entities that work for inclusive employment (different from the initial organizations with which the design was made) and, additionally, the conducting of group interviews with the managers of these organizations. This stage was orientated to assess both the relevance of the indicators and the possibility of implementation (in a short term), according to the level of "complexity" that each entity would experience.

The main techniques used during the stages of the study were literature review, documentary analysis and semi-structured interview. Even when the qualitative approach prevailed, numerical data analysis (valuation and weighing) was also performed in order to prioritize the indicators that would be part of the BSC.

As a main result of the project, a proposal for a Balanced Scorecard (BSC) was generated, with a high potential of implementation in the studied organizations. This tool included a set of 54 indicators, quantitative and qualitative, associated with 21 strategic objectives, mostly common in organizations that work for inclusive employment.

In general terms, the validation results of the model suggested that both the Strategic Map and the proposed BSC, are useful tools and highly adaptable to social entities that work for inclusive employment. However, the interviewed organizations suggested that the implementation should be carried out in a planned and gradual way, considering the resources and internal capacities of each entity. Thus, the tool was organized in three levels or stages of implementation so that the indicators could be adopted gradually according to measurement difficulties and associated costs. The levels were the following:

Level I: This is the basic version of the BSC. It includes 23 indicators that are easy to measure, since most organizations have the necessary data and information. It includes the "minimums" for elementary monitoring of strategic objectives. 
Level II: This intermediate level of the BSC, adds 20 indicators that require an "additional effort" to be measured, for example, conducting surveys, data analysis, reviewing external sources, etc.

Level III: This level, which represents the advanced version of the BSC, adds to the tool 11 indicators (impact or intangible), which require the use of qualitative techniques and the involvement of advanced measurement methods.

After presenting and evaluating the tool with organizations, it was also concluded that its adoption could result in various benefits for social entities, not only for the planning and controlling tasks, but also for the communicative and accountability duties. Nevertheless, it is necessary to advise that it is not entirely possible to "generalize" the model completely, even in organizations that have an almost identical mission (promotion of inclusive employment).

Although there is evidence of the adaptability of the objectives and indicators within some perspectives of the BSC, it is found that others could vary (or not apply) depending on the operation model of each entity and its own work. Therefore, it is recommended that before the implementation of the tool, the organization should make a review and, if necessary, an adaptation of the strategic map and its objectives, as well as, an assessment of the indicators, since these could be modified in accordance with the needs and priorities of a particular entity. The developed planning and monitoring instrument is sufficiently explanatory and flexible so that managers can adapt it according to their expectations, resources and capabilities.

The main value of this proposal is that, in addition to being based on a practical and proven planning and controlling methodology, it extracts and compiles other criteria proposed by recognized social evaluation approaches. The designed instrument includes a set of results and impact indicators that complement each other and contribute to a more comprehensive way to decision making in the social enterprise. The effectiveness and potential success of this BSC lies in the prioritization and selection of those indicators that are capable of adequately summarizing the organization's management, the change model and, specially, the generation of value for each stakeholder.

KEYWORDS: Balanced Scorecard, social impact, project evaluation, inclusive employment, social enterprise, social balance, social performance. 


\section{Introducción 1}

Los modelos de gestión orientados a resultados han ganado gran protagonismo en las últimas décadas. El concepto de Administración por Objetivos (en adelante APO), propuesto por Peter Drucker en su obra The practice of management (trad. 2012), sugiere un estilo de gerencia basado en el establecimiento de objetivos y su despliegue a cada área de la organización. Así, la APO busca comprometer a todos los colaboradores en el logro de las metas y resultados y, a su vez, exige por parte de los gerentes, actividades de autocontrol, control y medición para garantizar un adecuado desempeño y el cumplimiento del propósito organizacional (Drucker, 1986).

Este modelo administrativo, que busca crear un vínculo entre la planificación estratégica y la planificación operativa (Sánchez, 2003), es comúnmente adoptado por la empresa privada en su propósito de generar beneficios. Aunque en la entidad pública y en el tercer sector, métodos de este tipo no son tan extendidos, en los años recientes vienen ganando mayor popularidad. La búsqueda permanente de eficacia, eficiencia e impacto social es cada vez de mayor prioridad para la empresa no lucrativa, sus proveedores de recursos, donantes y demás grupos de interés.

Muchas de las entidades sociales y empresas sin ánimo de lucro, especialmente las más pequeñas, se enfrentan a una disyuntiva en el momento de decidir si aplican metodologías gerenciales fundadas originalmente para la empresa de producción o comercial con un claro objetivo económico. Los administradores de estas organizaciones temen que, al adoptar este tipo de modelos, puedan perder el "foco social" de su actuar y, por tanto, muestran resistencia a los mismos. Dicha renuencia, además, puede ser explicada por otros factores.

En primer lugar, no hay suficiente aceptación de las actividades de control y evaluación que la APO implica. La cultura organizacional que caracteriza una empresa social se diferencia por los valores de solidaridad y altruismo, por lo que se asume que todos los integrantes de la organización realizan las labores con eficacia y eficiencia -efecto de su indiscutible compromiso social-. A partir de estas premisas, se crea una percepción errada en los gestores, de que los procesos de control sobran y que incluso, "implantar métodos de gestión de ese trabajo no sólo sería superfluo, sino que podría ser interpretado como una señal de desconfianza hacia el equipo de colaboradores" (Austin, Gutierrez, Ogliastri y Reficco, 2006: 110).

1.- Una primera versión de este estudio se desarrolló como Trabajo Final de Máster (TFM) en el año 2016: Diseño de un cuadro de mando integral para la gestión del impacto social de entidades y proyectos sociales orientados al incremento del nivel de empleabilidad de colectivos en riesgo de exclusión. 
El segundo factor se asocia a las debilidades de planificación y gestión administrativa que muchas de estas organizaciones sociales poseen. De acuerdo con Curto (2012), es común que los gerentes o miembros de las juntas directivas no cuenten con el suficiente bagaje empresarial necesario para un direccionamiento exitoso y el logro de los objetivos de la empresa social, incluyendo el de sostenibilidad financiera. Tal y como lo planteo Leadbeater (1997), estos gestores requieren desarrollar habilidades ejecutivas y analíticas que les permita gerenciar entidades y proyectos más grandes y complejos.

La combinación de los objetivos de sostenibilidad y creación de beneficio social exige a las entidades sociales que trabajen de una manera más estratégica, lo que hasta el momento ha sido su talón de Aquiles. Los esfuerzos por actuar de manera urgente para solucionar problemáticas sociales y ejecutar acciones que impacten rápida y positivamente en los diferentes colectivos, han hecho que este tipo de organizaciones no destinen los suficientes recursos (y tiempo) a las actividades de planificación estratégica, medición y gestión del impacto social.

Un adecuado direccionamiento estratégico demanda una disciplina permanente de análisis del entorno cambiante y de las capacidades organizacionales. Sin embargo, en muchas de las entidades sociales (especialmente en las más pequeñas), este ejercicio se muestra algo limitado. En ocasiones, el proceso de planificación se reduce a una breve definición de conceptos como misión, visión, valores y objetivos, de manera básica y estática, y que solo revisten de relevancia a la hora de presentar la organización ante un tercero. La gestión estratégica debe contemplarse como un proceso más amplio y profundo, cíclico y dinámico. Para Hill y Jones (2010), este proceso contempla, además de la definición de la estrategia, otros elementos como la adopción de estructuras organizacionales y sistemas de control que garanticen su implantación.

Por la misma falta de profundidad en el proceso de planificación, y quizás por el afán de rendir cuentas a la comunidad, a los donantes y demás grupos de interés, muchas de las organizaciones sociales no logran total coherencia entre aquello que establecen como "estratégico", sus indicadores y sus sistemas de medición. Es común ver en el sector social como se plantean baterías de indicadores que buscan evidenciar ejecución de actividades 0 entrega de productos y servicios, pero que realmente sólo cumplen una misión informativa, y son escasos los que logran medir el impacto y la generación de valor.

Así pues, la evaluación o valoración del impacto social representa uno de los mayores retos dentro de la tarea de gestión y control de la empresa social. Como lo señala Díaz, Marcuello y Marcuello (2012), la medición del impacto social, frecuentemente está condicionada a la definición y aplicación de conjuntos de indicadores que ofrecen diversas entidades públicas o privadas, por ejemplo, la Auditoria Social, el Balance Social, el Global Reporting Initiative (GRI), etc. En medio de esta proliferación de propuestas se hace necesario, analizar, comparar, ajustar y, si es del caso, reconstruir modelos de evaluación que sean útiles para los administradores y financiadores de la empresa social. 
La dificultad para medir el valor social es indiscutible y reconocida por los gerentes, académicos y expertos. Evaluar el cumplimiento de la misión social de estas organizaciones, puede conllevar al desafío de medir nociones tan amplias y abstractas como el "alivio del sufrimiento humano" o la "protección de la biodiversidad" (Austin et al., 2006).

Además de que la cuantificación del impacto se considera uno de los aspectos más problemáticos -y por este solo hecho se vuelve absolutamente necesario involucrar enfoques cualitativos en su valoración-, la labor de evaluación puede ser aún más compleja, entre otros motivos por los siguientes: a) el desconocimiento de gestores y colaboradores sobre la cadena de resultados y las metodologías de medición, b) la información limitada y los altos costos en la recolección de datos, c) el cortoplacismo en las mediciones, d) la complejidad para medir intangibles ${ }^{2}$ y e) el conflicto en el establecimiento de la atribución ${ }^{3}$.

Con todo lo que hasta aquí se ha descrito, se puede resumir que la implementación de herramientas de monitoreo, evaluación y gestión del impacto social es una necesidad innegable para las organizaciones sin fines de lucro que trabajan por objetivos puramente sociales. El impacto social debe visibilizarse a fin de dar respuesta al actual contexto político, el mercado y la cooperación internacional, quienes continuamente incrementan sus niveles de exigencia frente a la eficacia de las intervenciones. Así, Bosma, Schott, Terjesen y Kew (2015), explican que el apremio de las empresas sociales por incursionar en procesos de medición de impacto, no solo responden a su deseo y necesidad de monitorear el cumplimiento de su objeto social, sino también a las presiones de inversores, donantes u otros grupos con especial interés en la generación de valor social y su visibilización.

En consecuencia, con la problemática, retos y desafíos explicados anteriormente, se establece como principal objetivo de este trabajo, diseñar una herramienta para la gestión del impacto social en organizaciones o proyectos sociales que trabajan en el campo del "empleo inclusivo". La propuesta se basa en una adaptación del Cuadro de Mando Integral (en adelante CMI) de Kaplan y Norton (1996). Se elige esta herramienta de planificación y control por su versatilidad y capacidad de aplicación a cualquier tipo de organización, además de que "ofrece un método más estructurado de selección de indicadores y esto permite que se produzca una mejor adaptación dentro de la gestión de la empresa" (Santos y Fidalgo, 2004: 4).

En el desarrollo de este trabajo se plantean además tres objetivos específicos: a) proponer una adaptación de conceptos teóricos relacionados al CMI según las características de la empresa social, b) definir los pilares de la planificación estratégica para las organizaciones o proyectos objeto de estu-

2.- Para efectos de este trabajo se aplica el concepto de intangibles en dos vías: a) para las personas, los intangibles serán entendidos como aquellas capacidades desarrolladas en el individuo que generan valor para él mismo y para su entorno (Murillo, 2016) y b) para las organizaciones, los intangibles representarán aquellos activos inmateriales que se constituyen en fuente de generación de valor de acuerdo a la estrategia y se centrarán, según la propuesta de Kaplan y Norton (2004), en el capital humano, capital de la información y capital organizacional. 3.- Medida que determina cuanto del impacto es causado por la contribución específica de un proyecto, intervención o servicio. 
dio y c) definir un conjunto de indicadores (de resultados y de impacto) que, asociados a los objetivos estratégicos, sirvan de guía para la medición y gestión del valor social.

El principal valor de la herramienta propuesta radica en que, además de basarse en un práctico y probado modelo de planificación y control, extrae y compila otros criterios que proponen reconocidos enfoques de evaluación social. El instrumento diseñado incluye un conjunto de indicadores de resultados e impacto que se complementan entre ellos y que pueden contribuir de una manera más integral a la toma de decisiones. La efectividad y éxito potencial de este CMI, se sustenta en la priorización y selección de aquellos indicadores que sean capaces de resumir adecuadamente la gestión de la organización, el modelo de cambio y, lo más importante, la generación de valor para cada grupo de interés (stakeholder).

Para el desarrollo del estudio se consideró la información aportada por dos entidades/proyectos vinculados a la Plataforma para el Cambio Social Nittua ${ }^{4}$ en el año 2016, y por dos fundaciones que tienen como misión mejorar la inserción laboral de colectivos en riesgo de exclusión. Estas dos fundaciones, específicamente, desarrollan su misión por medio de procesos de formación, intermediación laboral, acompañamiento y/o fomento del emprendimiento. Ambas, se encuentran ubicadas en la ciudad de Valencia (España) y tienen como principal público objeto de intervención, uno o varios de los siguientes colectivos: a) mujeres víctimas de violencia de género, b) emigrantes, c) personas con algún tipo de discapacidad física o intelectual y d) personas de edad avanzada en situación de desempleo.

Si bien, la construcción del mapa estratégico y del CMI se realizó con base a estas entidades (sus características, necesidades y capacidades internas), la metodología que se propone pretende ser lo suficientemente flexible para que pueda ser adaptada e implementada por otro tipo de organización o proyecto social.

Este documento se estructura de la siguiente manera: tras la introducción se presenta el marco teórico, donde se abordan los principales conceptos, modelos y enfoques que contribuyeron al desarrollo de este trabajo. En la sección tres se describe la metodología y las diferentes fases que se llevaron a cabo para la construcción del CMI. En la cuarta y quinta sección se presentan los resultados con su respectiva discusión y, finalmente, en la sección seis se recogen las principales conclusiones y recomendaciones.

4.- Nittúa es una organización social española que diseña, desarrolla, ejecuta y valora proyectos y herramientas que se constituyan en pilares para soportar el cambio social en la comunidad. Dentro de sus numerosas líneas de trabajo se destacan los proyectos en el campo de la inserción laboral de colectivos en riesgo de exclusión. 


\section{Marco teórico}

En este apartado se presenta, en primer lugar, el modelo de planificación y control sobre el cual se construye el instrumento de gestión de impacto social objeto de estudio (el mapa estratégico y el cuadro de mando integral). Posteriormente, se describen algunos enfoques y metodologías de evaluación de desempeño social, que fueron estudiadas para facilitar la definición de indicadores a incluir en la herramienta final.

\subsection{Mapa estratégico y Cuadro de Mando Integral (CMI)}

El Cuadro de Mando Integral y el Mapa Estratégico son modelos de gestión empresarial propuestos por los académicos Robert S. Kaplan y David P. Norton en sus obras The Balanced Scorecard: translating strategy into action (1996) y Strategy Maps: converting intangible assets into tangible outcomes (2004). El CMI se constituye en una herramienta de control y seguimiento, que haciendo uso de indicadores financieros y no financieros, evalúa el cumplimiento de la propuesta de valor establecida por una organización para sus diferentes grupos de interés (Kaplan y Norton, 1996).

El CMI no es únicamente un sistema de medición operativo. Aunado a un Mapa Estratégico, este cuadro de control puede considerarse como un sistema de gestión que busca transformar la visión, misión y estrategia de cualquier unidad de negocio en objetivos e indicadores tangibles. Tiene como característica esencial, que los empleados o colaboradores de cada área o proceso, entienden su responsabilidad sobre los resultados en el cumplimiento de la estrategia y, por tanto, asumen los compromisos en pro de los objetivos organizacionales. Como lo sugieren Méndez, Ayala y Palacio (2019), el CMI es una herramienta que permite a cualquier tipo de organización, equilibrar y gestionar todos los elementos que la componen y, asimismo, direccionar a sus equipos al cumplimiento de una visión compartida.

Kaplan y Norton (2004), plantean el Mapa Estratégico de un Cuadro de Mando Integral como una representación gráfica donde se establecen las relaciones causa-efecto entre los componentes de la estrategia organizacional (objetivos, actividades e indicadores) por medio de cuatro perspectivas básicas:

- Perspectiva financiera: Describe los resultados de la organización en términos económicos. Utiliza indicadores tradicionales como rentabilidad, crecimiento y productividad. Estos, representan los "resultados tangibles" orientados a evidenciar el valor que genera la organización para sus dueños o accionistas. 
- Perspectiva del cliente: Describe la propuesta de valor para los clientes o beneficiarios de los productos/servicios. Los indicadores que se definen en esta perspectiva deben dar cuenta del grado de cumplimiento de las expectativas y demandas del cliente y, por tanto, de su nivel de satisfacción.

- Perspectiva de los procesos internos: Identifica los procesos críticos que debe llevar a cabo la organización para cumplir con la propuesta de valor al cliente. Se define por aquellas decisiones "de operación" que impactan en la estrategia.

- Perspectiva de aprendizaje y crecimiento: Define los activos intangibles (capital humano, capital de la información y capital organizacional) necesarios para el desarrollo de la estrategia, el cumplimiento de la propuesta de valor al cliente y, como consecuencia, el logro de los objetivos financieros.

Los resultados asociados a cada una de las perspectivas deben estar conectados, alineados y balanceados, unos como facilitadores 0 inductores de otros, así: los resultados financieros serán viables en la medida en que se cumpla de manera exitosa la propuesta de valor del cliente (cliente satisfecho). Por otro lado, esta propuesta de valor podrá alcanzarse estableciendo procesos internos eficaces y eficientes los que, a su vez, requieren de capital humano y organizacional (activos intangibles) para operar de manera satisfactoria y garantizar el cumplimiento los objetivos estratégicos.

En la figura 1, se ilustra de una manera básica, la forma, contenidos e interacciones de un Cuadro de Mando Integral convencional.

\section{Figura 1. Cuadro de Mando Integral (Modelo Convencional)}

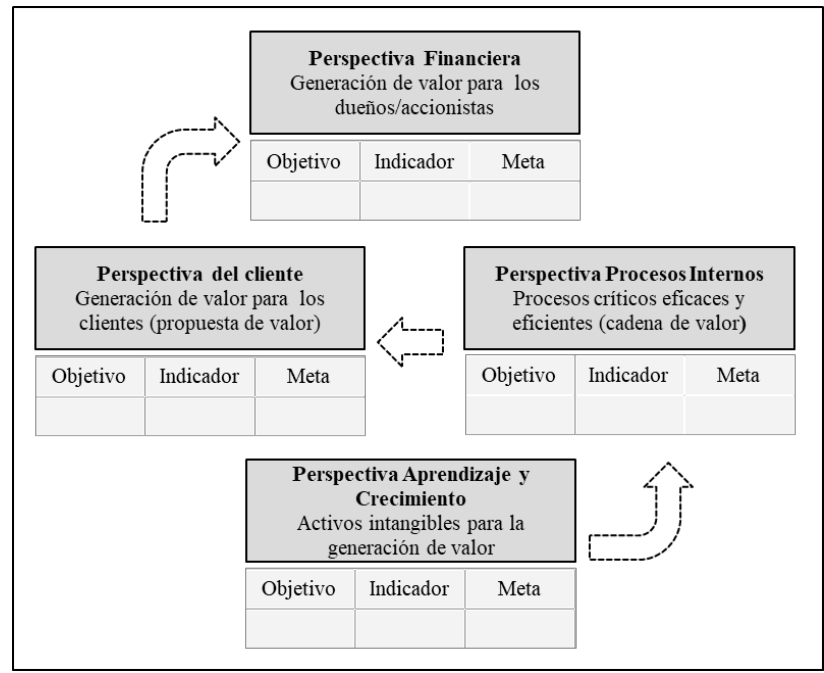

FUENTE: Elaboración propia basada en Kaplan y Norton (1996). 
Las relaciones de "causalidad" entre los elementos de la estrategia, son la principal característica del modelo CMI. Esta noción resulta especialmente importante para los gerentes, pues les permite tener un reflejo claro y sistémico de la estrategia del negocio y, por tanto, definir mejores formas de llevarla a cabo (Messeghem, Bakkali, Sammut y Swalhi, 2018). De esta manera, el CMI es frecuentemente usado como instrumento de gestión estratégica que busca convertir cada perspectiva en un conjunto de objetivos y metas claras, dando cabida a un sistema de evaluación que logra conectar la estrategia con las operaciones, tácticas y acciones de todas las personas y equipos de trabajo (Jahantigh, Malmir y Avilaq, 2018).

2.2. Aproximación de modelos lógicos para la evaluación del impacto social: la teoría del cambio y la cadena de resultados

La teoría de cambio puede considerarse como "un mapa semi-estructurado que enlaza nuestras acciones estratégicas a ciertos resultados de proceso que queremos provocar en nuestro entorno inmediato" (Retaloza, 2010: 4). Esta teoría, usada en diferentes contextos sociales y empresariales, consiste en definir una serie de hipótesis, las cuales, relacionadas de una forma causal, permitirán alcanzar un cambio deseado en un largo plazo.

La construcción de una teoría de cambio incluye la definición de: a) una visión de éxito, que describe una transformación a largo plazo; b) un conjunto de resultados previos, unos condicionantes de otros, que de manera causal facilitan el logro de la visión de éxito y c) un conjunto de supuestos que ayudan a sustentar y desarrollar la lógica de cambio. En palabras de Ortiz y Rivero, la teoría de cambio "explica cómo acciones consistentes, de manera lógica, predecible y probadamente resultarán en el cambio deseado" (2007, p.4). Este enfoque, presenta entonces una importante similitud con la propuesta de mapa estratégico. Ambas perspectivas consideran las relaciones causa-efecto como generadoras de cambio en un mediano o largo plazo.

La característica de "lógica causal" también es incorporada en el concepto de cadena de resultados. Según Gertler, Martínez, Premand, Rawlings y Vermeersch, la cadena de resultados es un modelo usado para describir una teoría de cambio, la cual "fija una definición lógica y plausible de cómo una secuencia de insumos, actividades y productos relacionados directamente con el proyecto interactúa con el comportamiento y define las vías para lograr los impactos" (2017: 38). 


\section{Figura 2. Cadena de resultados de un proyecto o intervención}

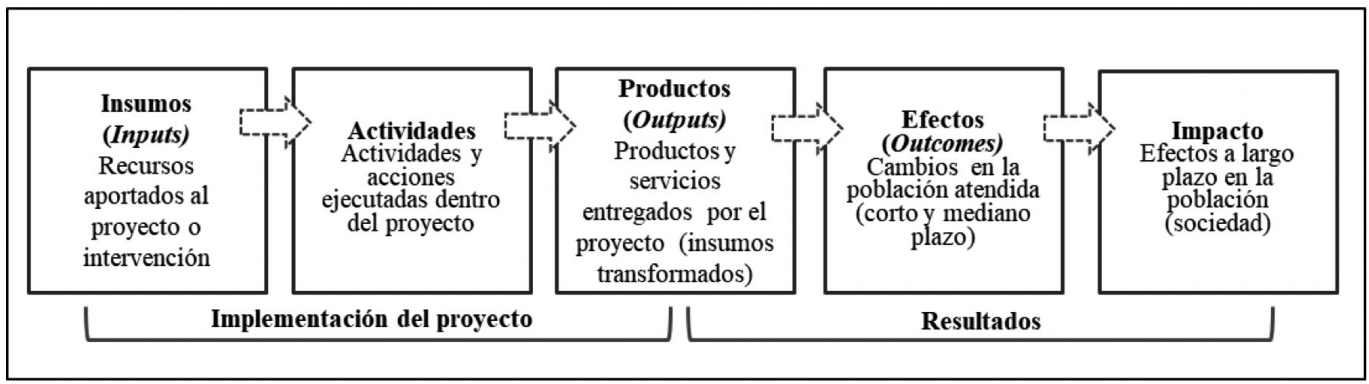

FUENTE: Elaboración propia basada en OECD (2002).

La cadena de resultados es una secuencia causal de unas etapas o elementos requeridos en el desarrollo de un proyecto o intervención a fin de alcanzar unos objetivos. Tal y como se ilustra en la figura 2, el inicio de la cadena lo establece los insumos (inputs), es decir, aquellos recursos financieros, humanos y materiales que serán transformados en productos (outputs) mediante las actividades. Los efectos (outcomes), son los cambios evidenciables a un corto o mediano plazo logrados por medio de los productos. Finalmente, se cierra la cadena con el impacto, el cual se define como el conjunto de efectos de largo plazo producidos por la intervención (OECD, 2002).

Con los mismos principios de los modelos lógicos descritos anteriormente, se han desarrollado, tanto los sistemas de evaluación y monitoreo de políticas públicas y programas de desarrollo (M\&E), como diversas metodologías y herramientas para la medición y valoración del impacto social. Dentro de estas metodologías, acogidas por entidades públicas, privadas y sociales, se destaca el Social Return On Investment (en adelante SROI).

El SROI tiene como objetivo medir los cambios que son importantes para las personas, organizaciones u otros grupos de interés, atribuibles a un proyecto o intervención. Esta herramienta se fundamenta en el concepto de valor social, entendido éste, como el conjunto de beneficios sociales, económicos y medioambientales para determinados grupos. Esta metodología propone un enfoque participativo y analítico, que permite capturar, en forma monetaria, un conjunto de cambios (pudiendo tener o no, valor en el mercado).

Así entonces, la medición SROI involucra un método de monetización ${ }^{5}$ y, consecuente a ello, produce un resultado expresado por medio de un ratio costo-beneficio. Dicho coeficiente describe cuantas unidades de moneda se generan en valor social por la inversión de una unidad de moneda.

5.- Asignación de valores monetarios (aproximaciones financieras) a las cosas que no necesariamente tienen valor en un mercado. 
Por ejemplo, un ratio de 3:1 sugiere que una inversión de 1 dólar, está generando un retorno de 3 dólares de valor social (Nicholls, Lawlor, Neitzer y Goodspeed, 2012).

Según Narrillos (2010), el proceso de monetización que propone el SROI podría generar varias ventajas para la organización, entre otras: a) facilita la compatibilización de los resultados financieros con los de gestión del valor, b) permite realizar análisis de sensibilidad, c) ayuda a identificar las fuentes de valor y d) facilita los procesos de comunicación con los grupos de interés que prefieren análisis cuantitativos. Adicionalmente a estos beneficios, que podrían materializarse o no (según la organización que evalúa, los grupos de interés y el contexto), es importante aclarar que la metodología SROI no se limita únicamente a un valor monetario, sino que, además, provee un marco sobre el cual se puede explorar cómo se genera el impacto social dentro de la organización (Rodríguez Díaz y Rey García, 2017).

Este marco o esquema sistémico, tiene sus bases en el denominado mapa de impacto o mapa de cambio, por medio del cual, se describe de manera cuantitativa y cualitativa la forma en la que se tangibiliza la cadena de resultados de la intervención y se genera valor para cada stakeholder. De aquí, resulta entonces un análisis integral que permite que los gerentes, inversores u otros grupos de interés, puedan tomar decisiones orientadas a la maximización del impacto de sus proyectos 0 inversiones.

2.3. El Balance Social: metodologías para la gestión y comunicación del desempeño social de la organización

El balance social se considera un instrumento de medición, auditoría, reporte y comunicación del desempeño social de una empresa u organización. Este puede ser implementado bajo la aplicación de una metodología específica que permita medir cuantitativa y cualitativamente la gestión social de la entidad (Colina y Senior, 2008). Las distintas metodologías de balance social, según Spear (2001), responden a uno, o varios, de los siguientes objetivos: a) realizar seguimiento al impacto social y ético de la organización, b) conformar una estrategia de gestión socialmente responsable, c) fomentar el aprendizaje organizacional para mejorar el desempeño social y d) comunicar a la comunidad y otros grupos de interés sobre la asignación y uso de los recursos.

Aunque no hay un método único para el uso e implementación de un balance social, dentro del contexto de la Responsabilidad Social Empresarial (RSE), existen diversas propuestas. Estas, por lo general, plantean un conjunto de criterios e indicadores asociados a los grupos de interés comunes en una organización. Para el desarrollo del presente trabajo, se abordan las siguientes metodologías: el Balance del Bien Común, el Global Reporting Initiative (en adelante GRI) y la Auditoría Social de REAS (Red de redes de economía alternativa y solidaria). 
El Balance del Bien Común es el pilar del modelo económico alternativo propuesto por Christian Felber en su obra La economía del bien común (2012). Este, promueve el desarrollo de un sistema de mercado donde las empresas no sobrepongan el ánimo de lucro como objetivo máximo de gestión, sino la contribución que puedan realizar al bien común y la cooperación. La Economía del Bien Común "coloca a los seres humanos y a todos los seres vivos, así como el éxito de las relaciones entre ellos, en el centro del sistema económico" (Moreno, 2012: 3).

El balance que propone Felber sienta sus bases en la denominada Matriz del Bien Común (MBC) ${ }^{6}$. Esta matriz, se compone de un conjunto de criterios de gestión, los cuales resultan de la interrelación de ciertos valores éticos universales y de las expectativas de los grupos de interés clave (y comunes) en toda empresa. Blachfellner et al. (2017) plantea esta herramienta como un modelo de desarrollo organizacional y de valoración, aclarando que "no se trata de una medición, sino de la aplicación de una escala de valores del Bien Común a las actividades concretas de una organización y el análisis de su impacto en los diferentes grupos de interés" (p. 8).

De otro lado se encuentra el Global Reporting Initiative (GRI), organización creada a finales de la década de los 90 que desarrolla guías de memorias de sostenibilidad orientadas a evidenciar la transparencia, desempeño e impacto social de todo tipo de entidades. Los referentes propuestos por el GRI contemplan criterios de información sobre la incidencia, positiva o negativa, que tienen las empresas en la economía, el medio ambiente y la sociedad (Global Reporting Initiative, 2013).

En el año 2013, el GRI emitió la Guía para las memorias de sostenibilidad G4 ${ }^{7}$ con el principal objetivo de apoyar a los gerentes de las empresas en la elaboración de unas memorias sólidas con información útil para la gestión y para los públicos de interés. Cinco años después, se realizó la transición de la guía G4 a los GRI Standards. Esta última versión se compone de una serie de estándares universales aplicables a cualquier tipo de organización (GRI 100), y otras tres series de estándares temáticos relacionados a impactos económicos, ambientales y sociales (GRI 200, 300 y 400 respectivamente). Sobre estos últimos, las organizaciones pueden seleccionar cuales usar en función de su sector y áreas de mayor impacto (Global Reporting Initiative, 2018).

6. - Se trabajó con la versión 4.1 de la MBC (y sus 17 indicadores) vigente en el año del desarrollo del proyecto (2016). Actualmente la matriz se encuentra en su versión 5.0, actualizada en el 2018.

7.- En este trabajo se utilizaron los criterios e indicadores de la versión G4 del GRI. Se realizó un análisis de los más de 60 indicadores, cuantitativos y cualitativos, y se hizo especial énfasis, en aquellos relacionados a la categoría de "desempeño social". 
El último modelo de balance social considerado en este trabajo, y especialmente reconocido en el sector de la economía solidaria española, es la Auditoría Social de REAS (Red de redes de economía alternativa y solidaria). Esta metodología tiene como principal objetivo:

(...) servir de herramienta de caracterización de las organizaciones de Economía Solidaria, medir su impacto social en función de sus objetivos y de los medios utilizados para conseguirlos y servir como proceso de aprendizaje que puede ser integrado en el ciclo normal de la planificación, seguimiento y evaluación de nuestras entidades. (Red de redes de economía alternativa y solidaria, 2018, párr. 2)

La auditoría social de REAS define un conjunto de indicadores, cuantitativos y cualitativos vinculados a seis principios de la economía solidaria (equidad, trabajo, sostenibilidad ambiental, cooperación, no fines lucrativos y compromiso con el entorno). Dichos indicadores también fueron analizados para la construcción del CMI objeto de estudio.

\subsection{El enfoque de capacidades y la medición del desarrollo}

De toda la gama de teorías desarrolladas en las últimas décadas referentes al bienestar y calidad de vida de las personas, una de las más relevantes es el enfoque de capacidades, propuesta por el nobel de economía Amartya Sen en su obra Desarrollo y Libertad (2000). Este enfoque postula una idea de desarrollo sujeta a la expansión del conjunto de capacidades (libertades) que un individuo tiene motivos para valorar y, así, obtener el grado de bienestar que desea en su vida. Sen, plantea que las capacidades se comportan como un fin del desarrollo y a la vez como medio para lograrlo.

Bajo este enfoque, el Programa de Naciones Unidas para el Desarrollo (PNUD) ha venido avanzando en el concepto de desarrollo humano, el cual ha definido como:

(...) un proceso en el cual se amplían las oportunidades. En un principio estas oportunidades pueden ser infinitas y cambiar con el tiempo. Sin embargo, a todos los niveles de desarrollo las tres más esenciales son disfrutar de una vida prolongada y saludable, adquirir conocimientos y tener acceso a los recursos necesarios para tener un nivel de vida decente". (PNUD, 1990: 34)

Consecuente con esto, el PNUD ha introducido en sus informes el Índice de Desarrollo Humano (IDH); indicador social que pretende evaluar el nivel de desarrollo de un país, haciendo hincapié en las personas y sus capacidades y no en el crecimiento económico per se. Si bien este índice refleja solo una parte de todas las capacidades que han de considerarse en el desarrollo humano, incluye tres dimensiones básicas: a) capacidad de tener una vida larga y saludable, medida según la esperanza de vida al nacer; b) capacidad de adquirir conocimientos, medida por los años promedio de escolaridad y c) capacidad de tener un nivel de vida digno, medida por el ingreso nacional bruto per cápita (PNUD, 2018). 
Las características anteriormente descritas sobre el enfoque de capacidades, sugieren que éste puede hacer un importante aporte a los esquemas de medición de impacto social de un proyecto 0 programa, entre otros motivos por los siguientes: a) se hace referencia al desarrollo desde el punto de vista multidimensional, b) se consideran las limitaciones personales, sociales y ambientales (factores de conversión) y c) se incluyen aspectos subjetivos, pero de gran valor, como lo es la percepción y satisfacción de las personas. Así lo confirma Garriga (2013):

(...) el enfoque de capacidades de Amartya Sen permite evaluar el bienestar y la calidad de vida con un mayor poder explicativo y comprensivo que cualquier enfoque basado en recursos, dinero, utilidad, satisfacción, bienes y derechos, siendo un marco de análisis óptimo para evaluar el bienestar producido por los programas (...). (p. 10)

\section{Metodología}

La investigación realizada fue de tipo descriptiva y práctica (o empírica). Mediante el análisis de la planificación estratégica de dos entidades/proyectos sociales y la revisión de importantes enfoques relacionados a la evaluación del desempeño o impacto social, se generó una propuesta de Cuadro de Mando Integral (CMI), con un alto potencial de aplicabilidad en las organizaciones estudiadas. Dicha propuesta busca contribuir a los procesos de planificación, control y evaluación de la empresa social.

Las principales técnicas utilizadas fueron la revisión bibliográfica, el análisis documental y la entrevista semiestructurada. Aun cuando predominó el enfoque cualitativo en el estudio, se realizó análisis de datos númericos (valoración y ponderación) a fin de priorizar los indicadores que harían parte del CMl.

La metodología empleada para el desarrollo de este trabajo incluyó cinco etapas, a saber: a) homologación de conceptos del CMI, b) elaboración del Mapa Estratégico, c) análisis y definición de indicadores, d) elaboración del CMl y e) validación y ajuste. A continuación, se presentan algunos aspectos importantes considerados en la selección de las organizaciones participantes, los procedimientos y métodos utilizados en las etapas anteriormente citadas:

Una vez homologados y adaptados los conceptos del mapa estratégico y el CMI, los cuales fueron pilares fundamentales para la construcción de la herramienta, se procedió a seleccionar dos organizaciones valencianas que estuvieran vinculadas a los proyectos de la Plataforma Nittúa, y sobre las cuales, posteriormente, se planteó el modelo inicial de planificación y medición (Mapa Estratégico 
y CMI). La elección de las organizaciones se realizó considerando los siguientes criterios: a) que la entidad o proyecto tuviera como misión u objetivo estratégico, el incremento de la empleabilidad de colectivos vulnerables y b) que la entidad o proyecto pudiera proveer información documentada sobre su estrategia y modo de operación.

Con las entidades/proyectos seleccionados y la información que estas aportaron en relación con su planificación y operación (objetivos, estrategias, acciones, metas, etc.), se procedió a examinar y analizar las directrices y elementos estratégicos que fueran comunes 0 de alta relevancia para cualquier organización que trabaje en pro del empleo inclusivo. Dichos elementos, posteriormente, dieron forma al mapa estratégico estándar.

Después de elaborado el mapa, se realizó una compilación de todos los indicadores asociados a los objetivos estratégicos de las organizaciones; haciendo uso de una matriz de análisis, se extractaron, contrastaron y analizaron, los indicadores provenientes de los modelos de evaluación social (descritos en el marco teórico), y los indicadores propuestos por las mismas entidades. De igual forma, se examinaron las relaciones entre estos indicadores, con cada una de las perspectivas del mapa estratégico y con los stakeholders involucrados.

A fin de determinar los indicadores más relevantes para incluir en el CMI, se llevó a cabo un proceso de ponderación, valoración y priorización, el cual permitió, reducir el número indicadores de 174 a 54. En la tabla 1, se describen los criterios y cálculos utilizados en esta depuración. La calificación y valoración de cada uno de los indicadores seleccionados puede ser consultado en la tabla del Apéndice A.

8.- El mapa estratégico "estándar" se refiere a un mapa estratégico general que podría ser adoptado por cualquier organización o proyecto que tenga como principal fin el "incremento de la empleabilidad en colectivos con riesgo de exclusión". 


\title{
Tabla 1. Criterios utilizados para la definición de indicadores
}

\author{
Criterio \\ Peso \\ Escala de calificación
}

C1: Aporte a la gestión del impacto:

Se evalúa que tanto aporte puede generar la medición y seguimiento del indicador a la gestión del impacto social o al monitoreo del objetivo estratégico asociado.
$50 \%$ Escala de 1 a 5, donde: 1 es "poco o nulo aporte a la gestión" y 5 es "gran aporte a la gestión"

\section{C2: Facilidad y costo de la medición:}

Se evalúa que tan "factible" podría ser la medición del indicador considerando: a) disponibilidad de información, b) 30\% métodos de medición, c) costo de recolección de datos y d) capacidades internas de la organización
Escala de 1 a 5, donde: 1 es "medición muy costosa y/o muy complicada" y 5 es "de muy fácil medición y sin costos altos adicionales"
C3: Involucramiento de los stakeholders:

Se valora que tantos grupos de interés se están beneficiando de la información o están involucrados en la variable central del indicador (sobre un total de cinco grupos de interés identificados).
Escala de 1 a 5, donde: 1 es "uno de los grupos de interés establecidos en la matriz está relacionado

$20 \%$ al indicador" y 5 es "cinco de los grupos de interés establecidos en la matriz están relacionados al indicador"

\section{Valoración y priorización}

La valoración final de cada indicador resulta de la sumatoria de la calificación de cada criterio por el peso porcentual respectivo, así;

Valoración indicador $\mathbf{X}=\left(\right.$ Calificación $\left.\mathrm{C} 1^{*} 0,5\right)+\left(\right.$ Calificación $\left.\mathrm{C} 2^{*} 0,3\right)+\left(\right.$ Calificación $\left.\mathrm{C} 3^{*} 0,2\right)$

Se establecen como prioritarios, aquellos indicadores cuya valoración haya sido superior a 3,7.

\section{FUENTE: Elaboración propia.}

Con el mapa estratégico definido y los indicadores priorizados, se dio paso al diseño y construcción del CMI, objeto del estudio. La validación final de la propuesta y sus respectivos indicadores se realizó con la participación de dos entidades sociales valencianas que trabajan por el empleo inclusivo (diferentes a las entidades iniciales con las cuales se trabajó el diseño).

Por medio de entrevistas y sesiones de trabajo con los gestores de estas dos fundaciones, se validó la herramienta, analizando específicamente los siguientes elementos: a) la capacidad de adaptación del mapa estratégico y el CMI propuesto a la organización, b) la pertinencia de los indicadores para la gestión y posibles dificultades en la medición de los mismos y c) el grado de aporte que podría generar la medición y el control de los indicadores para la toma de decisiones estratégicas orientadas a la maximización del impacto social. 
Es necesario aclarar que el proceso de validación se limitó a la presentación del modelo y a la realización de entrevistas grupales en cada una de las entidades donde, además de indagar sobre la pertinencia de cada indicador, también se evaluó su posibilidad de implementación (en un corto plazo) según el grado de "complejidad" que para cada entidad representaría. Dicha complejidad fue analizada en función de los siguientes factores: a) el costo adicional que implicaría para la organización (tiempo y recursos), b) las estadísticas e información documentada disponible en la actualidad y c) el conocimiento del equipo en metodologías necesarias para llevar a cabo evaluaciones de impacto.

Por último, teniendo en cuenta los resultados de esta validación, se procedió a elaborar cambios y adaptaciones a algunos de los indicadores o partes del modelo. Adicionalmente se llevó a cabo un proceso de clasificación, en el cual, se asignaron diferentes niveles a cada indicador de acuerdo al grado de dificultad en su medición. Así, finalmente, se proyectó la herramienta (CMI final) en tres niveles o versiones: básico, intermedio y avanzado.

\section{Resultados}

\subsection{Adaptación de conceptos del CMI a la organización social}

El primer producto que arrojó este estudio, y que a su vez se constituyó en base para el logro de los objetivos subsiguientes, fue la adaptación de conceptos del CMI de acuerdo con las características de las organizaciones sociales y según la terminología o lenguaje organizacional que en este sector predomina. Una vez analizados los modelos propuestos por Kaplan y Norton, se realizó una homologación de términos, con el fin de que los elementos centrales de un Mapa Estratégico y un $\mathrm{CMI}$, puedan ser asimilados y mejor aplicados por las organizaciones sociales que trabajan para la mejora del empleo inclusivo. Esta adaptación, se presenta en la tabla 2. 


\section{Tabla 2. Adaptación de conceptos teóricos del Mapa Estratégico y el CMI para las entidades sociales}

\begin{tabular}{|c|c|c|}
\hline Concepto & Empresa convencional & Entidad o proyecto social \\
\hline Estrategia & $\begin{array}{l}\text { Forma de actuación con el fin de crear valor para } \\
\text { accionistas y clientes }\end{array}$ & $\begin{array}{l}\text { Forma de actuación con el fin de generar valor para los } \\
\text { beneficiarios y demás stakeholders }\end{array}$ \\
\hline $\begin{array}{l}\text { Creación de } \\
\text { Valor }\end{array}$ & $\begin{array}{l}\text { Aumento de valor para los accionistas mediante el } \\
\text { crecimiento de los ingresos y la productividad }\end{array}$ & $\begin{array}{l}\text { Generación de impacto social y satisfacción de las } \\
\text { necesidades de todos los beneficiarios de las intervenciones } \\
\text { y demás stakeholders }\end{array}$ \\
\hline $\begin{array}{l}\text { Perspectivas } \\
\text { del CMI }\end{array}$ & $\begin{array}{l}\text { 1) Financiera, 2) Clientes, 3) Procesos internos y 4) } \\
\text { Aprendizaje y crecimiento }\end{array}$ & $\begin{array}{l}\text { 1) Social, 2) Sostenibilidad Financiera, 3) Procesos internos y } \\
\text { 4) Aprendizaje y crecimiento. }\end{array}$ \\
\hline Cliente & $\begin{array}{l}\text { Persona u organización que paga por el producto } 0 \\
\text { servicio }\end{array}$ & $\begin{array}{l}\text { 1) Persona u organización que paga o aporta a la prestación } \\
\text { del servicio o intervención (donante, cooperante) y 2) } \\
\text { Persona o grupo de personas que se benefician del servicio } \\
\text { (usuarios, familia, sociedad) }\end{array}$ \\
\hline $\begin{array}{l}\text { Perspectiva } \\
\text { financiera }\end{array}$ & $\begin{array}{l}\text { Máxima visión de éxito y nivel jerárquico superior del } \\
\text { CMI. Establece objetivos y estrategias orientadas } \\
\text { a la maximización de beneficios e incremento de } \\
\text { rentabilidad }\end{array}$ & $\begin{array}{l}\text { Denominada "Sostenibilidad Financiera". No representa } \\
\text { el último fin de la entidad, sino un medio para alcanzar el } \\
\text { impacto social deseado (perspectiva social). Establece } \\
\text { objetivos orientados a la gestión responsable de recursos. }\end{array}$ \\
\hline $\begin{array}{l}\text { Perspectiva } \\
\text { del cliente }\end{array}$ & $\begin{array}{l}\text { Ocupa el segundo nivel del CMI. Se enfoca en la } \\
\text { propuesta de valor al cliente, la cual inducirá al logro } \\
\text { de los objetivos financieros }\end{array}$ & $\begin{array}{l}\text { Denominada "Perspectiva Social". Ocupa, junto con la } \\
\text { perspectiva de "Sostenibilidad Financiera", el nivel jerárquico } \\
\text { superior del CMl. Aquí se establece su máxima visión de } \\
\text { éxito: Impacto Social }\end{array}$ \\
\hline $\begin{array}{l}\text { Perspectiva } \\
\text { procesos } \\
\text { internos }\end{array}$ & $\begin{array}{l}\text { Conjunto de actividades/procesos críticos que definen } \\
\text { la "cadena de valor" (desarrollo de la propuesta del } \\
\text { valor al cliente) }\end{array}$ & $\begin{array}{l}\text { Conjunto de actividades críticas necesarias para el desarrollo } \\
\text { de la intervención o la prestación del servicio a la comunidad } \\
\text { y colectivos beneficiarios. }\end{array}$ \\
\hline $\begin{array}{l}\text { Perspectiva } \\
\text { aprendizaje y } \\
\text { crecimiento }\end{array}$ & $\begin{array}{l}\text { Describe los objetivos relacionadas al uso y } \\
\text { maximización de los activos intangibles de la } \\
\text { organización (capital humano, capital de la } \\
\text { información, capital organizacional) }\end{array}$ & $\begin{array}{l}\text { Describe los objetivos y estrategias orientadas al desarrollo } \\
\text { de capacidades en los colaboradores de la organización y } \\
\text { en la propia entidad (formación, gestión del conocimiento, } \\
\text { comunicación, clima laboral, etc.) }\end{array}$ \\
\hline $\begin{array}{l}\text { Lógica } \\
\text { del mapa } \\
\text { estratégico y } \\
\text { del CMI }\end{array}$ & $\begin{array}{l}\text { Desde el nivel jerárquico superior, se establece } \\
\text { la hipótesis de que los resultados financieros } \\
\text { (perspectiva financiera) sólo pueden conseguirse si } \\
\text { los clientes están satisfechos (perspectiva del cliente). } \\
\text { Los procesos internos alineados con los activos } \\
\text { intangibles permiten el desarrollo de la propuesta de } \\
\text { valor para el cliente }\end{array}$ & $\begin{array}{l}\text { Desde el nivel jerárquico superior, se establece la hipótesis } \\
\text { de que los objetivos sociales (perspectiva social) sólo pueden } \\
\text { conseguirse si: 1) se logra una adecuada administración de } \\
\text { los recursos (perspectiva sostenibilidad financiera) y 2) los } \\
\text { procesos internos y los activos intangibles se encuentran } \\
\text { alineados y funcionan en pro de los objetivos sociales y } \\
\text { financieros. Adicionalmente se destacan las relaciones } \\
\text { causa-efecto en doble vía, entre las perspectivas social y } \\
\text { de sostenibilidad financiera, pues la captación de recursos } \\
\text { puede verse condicionada a los resultados sociales } \\
\text { demostrables para los donantes o cooperantes. }\end{array}$ \\
\hline
\end{tabular}

FUENTE: Elaboración propia con información de Kaplan y Norton (2004). 


\subsection{Elaboración del Mapa Estratégico estándar}

Posterior a la adaptación y comprensión de los conceptos se construyó un esquema de mapa estratégico estándar, en el cual, se estableció como objetivo primordial (misión) el "incremento del nivel de empleabilidad de los colectivos en riesgo de exclusión". Este mapa, ilustrado de manera resumida en la figura 3 , incluyó dentro de cada una de sus perspectivas, aquellos objetivos estratégicos transversales o relevantes identificados en la planificación de las organizaciones analizadas. Estos, a su vez, se convirtieron en los pilares del diseño del Cuadro de Mando Integral.

\section{Figura 3. Mapa Estratégico estándar para entidades /proyectos de empleo inclusivo}

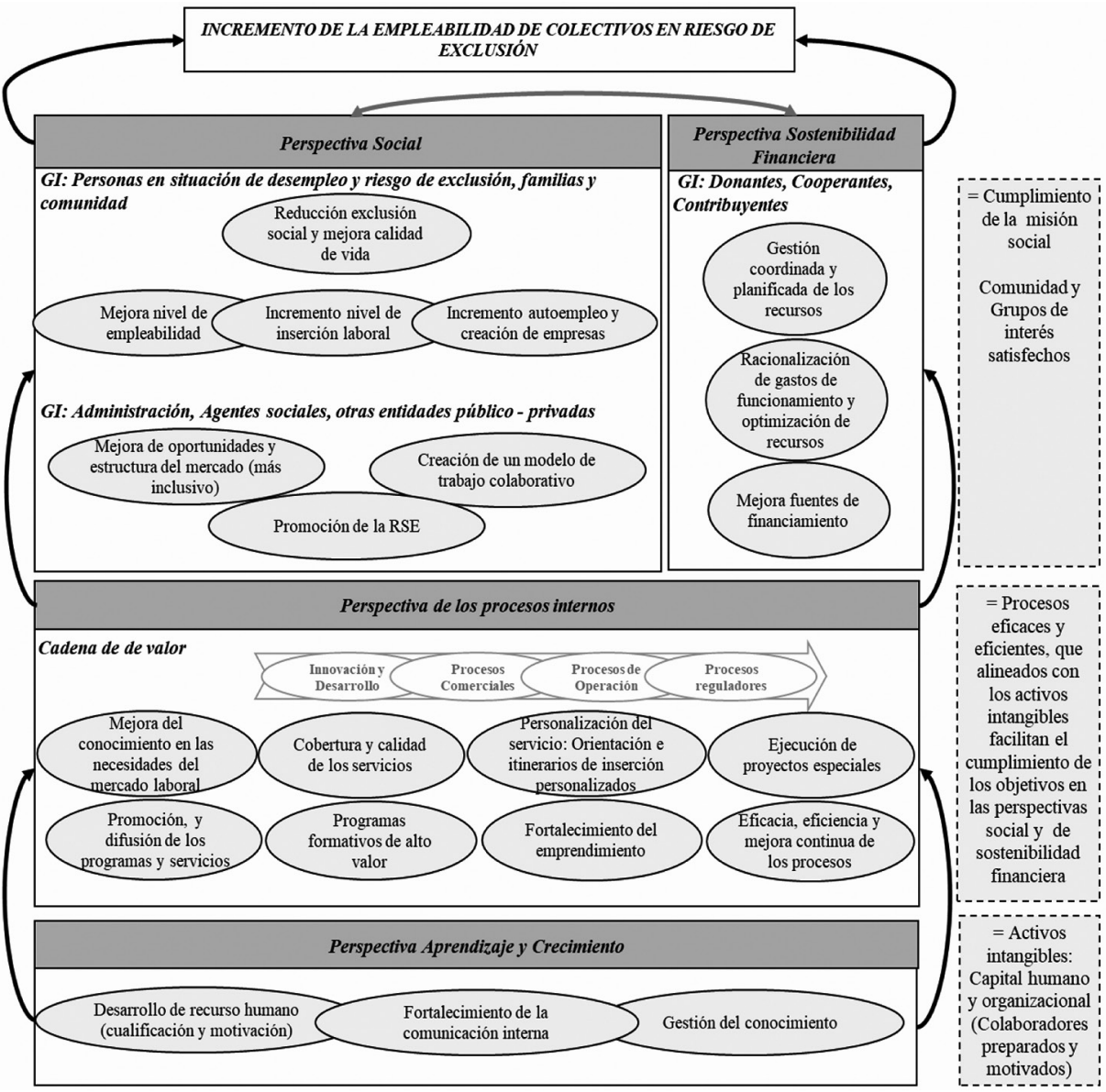

FUENTE: Elaboración propia basada en Murillo (2016). 


\subsection{Selección de indicadores y diseño del Cuadro de Mando Integral}

Una vez realizado el proceso de depuración y valoración de los indicadores, se procedió a diseñar la primera propuesta del Cuadro de Mando Integral (CMI) que incluyó un total de 54 indicadores (cuantitativos y cualitativos). Estos, fueron clasificados según el objetivo estratégico y la perspectiva asociada (figura 3). En la tabla 3, se presenta el número de indicadores resultantes por cada una de las perspectivas y objetivos estratégicos y, en el apéndice $A$, se puede consultar el listado completo de todos los indicadores con su respectiva caracterización.

\section{Tabla 3. Número de indicadores del CMI por perspectiva y objetivo}

\begin{tabular}{|c|c|c|}
\hline Objetivo estratégico & $\begin{array}{c}\mathrm{N}^{\circ} \text { indicadores } \\
\text { cuantitativos }\end{array}$ & $\begin{array}{c}N^{\circ} \text { indicadores } \\
\text { cualitativos }\end{array}$ \\
\hline \multicolumn{3}{|l|}{ Perspectiva Social } \\
\hline Reducción exclusión social y mejora calidad de vida & 5 & 4 \\
\hline Mejora nivel de empleabilidad & 2 & 1 \\
\hline Incremento nivel de inserción laboral & 2 & 0 \\
\hline Incremento autoempleo y creación de empresas & 1 & 0 \\
\hline Mejora de oportunidades y estructura del mercado laboral (más inclusivo) & 1 & 1 \\
\hline Creación de modelos de trabajo colaborativos & 1 & 1 \\
\hline Promoción de la Responsabilidad Social Empresarial (RSE) & 3 & 2 \\
\hline \multicolumn{3}{|c|}{ Perspectiva Sostenibilidad Financiera } \\
\hline Mejora de fuentes de financiamiento & 3 & 0 \\
\hline Racionalización de gastos de funcionamiento y optimización de recursos & 3 & 0 \\
\hline Gestión coordinada y planificada de los recursos & 2 & 0 \\
\hline \multicolumn{3}{|c|}{ Perspectiva Procesos Internos } \\
\hline Mejora del conocimiento sobre las necesidades del mercado laboral & 0 & 1 \\
\hline Cobertura y calidad de los servicios & 4 & 0 \\
\hline Promoción y difusión de los programas y servicios & 1 & 0 \\
\hline Personalización del servicio (itinerarios de inserción personalizados) & 1 & 0 \\
\hline Programas de alto valor formativo & 1 & 0 \\
\hline Fortalecimiento del emprendimiento & 2 & 0 \\
\hline Eficacia, eficiencia y mejora continua de los procesos & 2 & 0 \\
\hline Ejecución de proyectos especiales & 1 & 1 \\
\hline \multicolumn{3}{|c|}{ Perspectiva de aprendizaje y crecimiento } \\
\hline Desarrollo del recurso humano (cualificación y motivación) & 4 & 1 \\
\hline Fortalecimiento de la comunicación interna & 1 & 1 \\
\hline Gestión del conocimiento & 0 & 1 \\
\hline
\end{tabular}

FUENTE: Elaboración propia. 


\section{CUADRO DE MANDO INTEGRAL PARA LA GESTIÓN DEL IMPACTO SOCIAL EN ORGANIZACIONES DE EMPLEO INCLUSIVO}

El CMI diseñado consistió en un esquema sencillo, pero a la vez descriptivo, que tuvo como fin proveer a las entidades sociales y a sus gestores una herramienta que les permitiera visualizar y controlar el logro gradual de sus objetivos estratégicos. Con los indicadores seleccionados se construyó el instrumento usando una hoja de cálculo de fácil manejo y entendimiento. Como se observa en la figura 5, dicha herramienta, además, incluyó un elemento gráfico condicional (semáforo) que permite al usuario visualizar de una manera rápida, donde se generan alertas por el nivel de cumplimiento según las metas que se establezcan para cada indicador.

\section{Figura 4. Ejemplo modelo CMI (extracto de la perspectiva social)}

\begin{tabular}{|c|c|c|c|c|c|c|c|c|c|c|c|c|}
\hline \multicolumn{12}{|c|}{ PERSPECTIVA SOCIAL } & \multirow[b]{2}{*}{$\mathbf{N}$} \\
\hline $\begin{array}{l}\text { Obietivo / Indicador } \\
\text { a medir }\end{array}$ & Definición indicador & Dimensión & $\mathbf{T}$ & İndice / Descripción & Frecuencia & Fuente & Responsable & Meta & Periodo & Resultado & Alerta & \\
\hline \multirow{2}{*}{$\begin{array}{l}\text { Nivel de } \\
\text { satisfacción }\end{array}$} & $\begin{array}{l}\text { Porcentaje de beneficiarios(as) que se } \\
\text { consideran "Satisfechos" o "Muy satisfechos" } \\
\text { con los servicios recibidos, la intervención o } \\
\text { proyecto. }\end{array}$ & Cuantitativo & $\mathrm{R}$ & $\begin{array}{c}\text { ( } \mathrm{N}^{\circ} \text { de beneficiarios que expresan en la } \\
\text { encuesta de satisfacción estar "Satisfechos" } \\
\text { o "Muy satisfechos" con el servicio / Total de } \\
\text { beneficiarios encuestados) * } 100\end{array}$ & Mensual & $\begin{array}{l}\text { Encuesta de } \\
\text { satisfacción }\end{array}$ & Gestor X & $100 \%$ & Ene Jun 16 & $100 \%$ & 0 & $\|$ \\
\hline & $\begin{array}{l}\text { Percepción de los(a) beneficiarios en cuanto a } \\
\text { la satisfacción de sus necesidades, } \\
\text { resolución de problemas y expectativas }\end{array}$ & Cualitativo & 1 & Informe cualitativo & Semestral & $\begin{array}{l}\text { Encuesta de } \\
\text { satisfacción }\end{array}$ & Gestor X & Positivo & Ene-Jun 16 & Posifiva & & $\|$ \\
\hline $\begin{array}{l}\text { Variación de la } \\
\text { renta familiar }\end{array}$ & $\begin{array}{l}\text { Porcentaje de beneficiarios(as) que } \\
\text { incrementan el valor de su renta familiar en los } \\
\text { últimos dos años. }\end{array}$ & Cuantitativo & 1 & $\begin{array}{l}\text { (No de beneficiarios que demuestran o } \\
\text { informan incremento de la renta familiar / } \\
\text { Total de beneficiarios evaluados) })^{*} 100\end{array}$ & $>1$ aก̂。 & $\begin{array}{l}\text { Línea de Base } \\
\text { Evaluación } \\
\text { Expost }\end{array}$ & Gestor $Y$ & $50 \%$ & $2014-2016$ & $49 \%$ & & III \\
\hline \multirow{2}{*}{$\begin{array}{l}\text { Calidad de la } \\
\text { vivienda }\end{array}$} & $\begin{array}{l}\text { Porcentaje de beneficiarios(as) que adquieren } \\
\text { vivienda o mejoran la calidad de la misma en } \\
\text { los últimos dos años. }\end{array}$ & Cuantitativo & 1 & $\begin{array}{l}\text { ( } \mathrm{N}^{\circ} \text { de beneficiarios que demuestran } 0 \\
\text { informan mejora en la calidad de la vivienda / } \\
\text { Total de beneficiarios evaluados) * } 100\end{array}$ & $>1$ año & $\begin{array}{c}\text { Línea de Base } \\
\text { Evaluación } \\
\text { Expost }\end{array}$ & Gestor $Y$ & $30 \%$ & $2014-2016$ & $10 \%$ & 0 & III \\
\hline & $\begin{array}{l}\text { Percepción de los(a) beneficiarios en cuanto a } \\
\text { la mejora de la calidad de su vivienda y la } \\
\text { contribución del servicio o proyecto en este }\end{array}$ & Cualitativo & 1 & Informe cualitativo & $>1$ aก̃o & $\begin{array}{c}\text { Línea de Base } \\
\text { Evaluación } \\
\text { Expost }\end{array}$ & Gestor $Y$ & Positivo & $2014-2016$ & Negativo & & III \\
\hline \multirow{2}{*}{$\begin{array}{l}\text { Calidad de la } \\
\text { salud }\end{array}$} & $\begin{array}{l}\text { Porcentaje de beneficiarios(as) que mejoran la } \\
\text { calidad de su salud en los últimos dos años. }\end{array}$ & Cuantitativo & 1 & $\begin{array}{l}\text { ( } \mathrm{N}^{\circ} \text { de beneficiarios que demuestran } 0 \\
\text { informan mejora en la calidad de la salud / } \\
\text { Total de beneficiarios evaluados) * } 100\end{array}$ & $>1$ aก̃o & $\begin{array}{l}\text { Linea de Base } \\
\text { Evaluación } \\
\text { Expost }\end{array}$ & Gestor $Y$ & $30 \%$ & $2014-2016$ & $30 \%$ & 0 & III \\
\hline & $\begin{array}{l}\text { Percepción de los(a) beneficiarios en cuanto a } \\
\text { la mejora de la calidad de su salud y la } \\
\text { contribución del servicio o proyecto en este }\end{array}$ & Cualitativo & 1 & Informe cualitativo & $>1$ aก̃o & $\begin{array}{c}\text { Línea de Base } \\
\text { Evaluación } \\
\text { Expost }\end{array}$ & Gestor $Y$ & Positivo & $2014-2016$ & Positivo & & III \\
\hline \multirow{2}{*}{\begin{tabular}{|l|} 
Incremento de la \\
autonomia
\end{tabular}} & $\begin{array}{l}\text { Porcentaje de beneficiarios(as) que mejoran la } \\
\text { su autonomía en los últimos dos años, } \\
\text { entendida esta como la capacidad del } \\
\text { individuo (fisica, mental e intelectual) para } \\
\text { desarrollar las actividades básicas de la vida }\end{array}$ & Cuantitativo & 1 & $\begin{array}{l}\left(\mathrm{N}^{\circ} \text { de beneficiarios que demuestran o }\right. \\
\text { informan mejora en su autonomia / Total de } \\
\text { beneficiarios evaluados) * } 100\end{array}$ & $>1$ año & $\begin{array}{l}\text { Línea de Base } \\
\text { Evaluación } \\
\text { Expost }\end{array}$ & Gestor $Y$ & $40 \%$ & $2014-2016$ & $38 \%$ & & III \\
\hline & $\begin{array}{l}\text { Percepción de los(a) beneficiarios en cuanto } \\
\text { al incremento de su autonomía para el } \\
\text { desarrollo de las actividades básicas de la }\end{array}$ & Cualitativo & 1 & Informe cualitativo & $>1$ aก̃o & $\begin{array}{l}\text { Línea de Base } \\
\text { Evaluación } \\
\text { Expost }\end{array}$ & Gestor $Y$ & Positivo & $2014-2016$ & Pasitivo & & III \\
\hline Nivel educativo & $\begin{array}{l}\text { Porcentaje de beneficiarios(as) que se } \\
\text { alfabetizan y/o incrementan su nivel educativo } \\
\text { en los últimos dos años. }\end{array}$ & Cuantitativo & 1 & $\begin{array}{l}\left(\mathrm{N}^{\circ} \text { de beneficiarios que demuestran o }\right. \\
\text { informan incremento de su nivel educativo/ } \\
\text { Total de beneficiarios evaluados) }{ }^{*} 100\end{array}$ & $>1$ año & $\begin{array}{l}\text { Línea de Base } \\
\text { Evaluación } \\
\text { Expost }\end{array}$ & Gestor $Y$ & $40 \%$ & $2014-2016$ & $40 \%$ & 0 & $\|$ \\
\hline \multirow{2}{*}{$\begin{array}{l}\text { Cualificación } \\
\text { profesional I } \\
\text { laboral }\end{array}$} & $\begin{array}{l}\text { Porcentaje de beneficiarios(as) que } \\
\text { incrementan su nivel de competencias } \\
\text { laborales en el último año. }\end{array}$ & Cuantitativo & 1 & $\begin{array}{l}\text { ( } \mathrm{N}^{\circ} \text { de beneficiarios que demuestran o } \\
\text { informan incremento de sus competencias } \\
\text { laborales / Total de beneficiarios evaluados) } \\
{ }_{100}\end{array}$ & Anual & $\begin{array}{c}\text { Linea de Base } \\
\text { Evaluación } \\
\text { Expost }\end{array}$ & Gestor $Y$ & $70 \%$ & $2014-2016$ & $64 \%$ & 0 & $\|$ \\
\hline & $\begin{array}{l}\text { Percepción de los(a) beneficiarios en cuanto } \\
\text { al incremento de sus competencias laborales } \\
\text { y la contribución del servicio o proyecto en }\end{array}$ & Cualitativo & 1 & Informe cualitativo & $>1$ aก̃o & $\begin{array}{c}\text { Línea de Base } \\
\text { Evaluación } \\
\text { Expost }\end{array}$ & Gestor $Y$ & Positivo & $2014-2016$ & Negativa & & $\|$ \\
\hline
\end{tabular}

T: clasifica el indicador según el tipo de medición; indicador de resultados a corto plazo (R) o indicador de impacto (I). Las columnas "Frecuencia", "Fuente", "Responsable", "Meta", "Periodo" describen los parámetros que cada organización considere pertinentes, de acuerdo a su planificación estratégica y operativa (los datos contenidos en dichas columnas se registran a manera de ejemplo).

FUENTE: Elaboración propia. 


\subsection{Validación del Cuadro de Mando Integral y ajustes}

En general, los resultados de la validación del modelo sugirieron que tanto el Mapa Estratégico, como el CMl propuesto, son herramientas de utilidad y con alto potencial de ser adaptadas y utilizadas por las organizaciones sociales que trabajan por el empleo inclusivo. Sin embargo, fue común el sentir de que el proceso de adaptación e implementación del CMI debe desarrollarse de manera planificada y paulatina.

Debido a la limitada cultura de control y a los esfuerzos "adicionales" que significa para las organizaciones realizar actividades de medición, estas, se muestran temerosas o reacias a la hora de plantearse algunos de los indicadores propuestos (especialmente de impacto). Por este motivo, se consideró que la mejor manera de implementar la herramienta es adoptando los indicadores de forma escalonada según el grado de "complejidad" en su medición y los costos asociados a la misma. Así pues, se proyecta el CMI, en tres niveles de implementación:

Nivel I: Contiene 23 indicadores que conforman el CMI en su versión básica. Estos indicadores son fáciles de medir, ya que las organizaciones, en su mayoría, cuentan con la información aunque no sea de forma ordenada o documentada. Comprende los "mínimos" para hacer un seguimiento elemental a los objetivos estratégicos del mapa.

Nivel II: Agrega al modelo 20 indicadores que requieren de un "esfuerzo adicional" para su medición, por ejemplo, la aplicación de encuestas, extracción y análisis de datos, consulta de fuentes externas, etc. Este nivel se constituye en la versión intermedia del CMI.

Nivel III: Incluye la totalidad de los indicadores propuestos. Este nivel, que representa la versión avanzada del CMI, agrega a la medición 11 indicadores (de impacto o de intangibles), los cuales requieren la utilización de técnicas cualitativas y el involucramiento de otros métodos más avanzados de medición y valoración, como lo es el SROI, las líneas de base y las evaluaciones ex post en un largo plazo.

En el apéndice A, se señala el nivel de implementación al que corresponde cada uno de los indicadores, según su dificultad de medición. 


\section{Discusión}

El Cuadro de Mando Integral (CMI) adaptado a las entidades sociales resulta en una herramienta de gestión de interés para sus gerentes y gestores, pues se constituye en un instrumento de monitoreo permanente al logro de los objetivos estratégicos que puede facilitar y soportar la toma de decisiones. Al igual que el modelo convencional del Balanced Scorecard de Kaplan y Norton (1996), la herramienta propuesta traduce la misión de la organización (empleo inclusivo) en un conjunto de indicadores, cuantitativos y cualitativos, financieros y no financieros, que reflejan el equilibrio entre los objetivos de corto y largo plazo establecidos dentro de la estrategia.

Aproximadamente, el 26\% de los indicadores incluidos dentro del CMI son de carácter cualitativo y están principalmente vinculados a los objetivos de las perspectivas social y de aprendizaje y crecimiento. Lo cual se debe a que la evaluación y análisis del cumplimiento de los objetivos de estas dos perspectivas, implica la "medición" de resultados más intangibles, que sean capaces de describir la evolución de las capacidades personales y organizacionales y aquellos cambios de más largo plazo que la intervención podría estar generando en las familias, la comunidad, los empleados y otros grupos de interés.

El esquema de medición resultante, concebido desde la construcción de un Mapa Estratégico que sigue la lógica causal "Misión - Perspectiva - Objetivo Estratégico - Indicador", ofrece a las organizaciones sociales, una metodología concreta para la planificación estratégica, operativa y para los procesos de control y evaluación. Esta herramienta, excluye mediciones innecesarias no generadoras de valor en la toma de decisiones y, por consiguiente, puede considerarse más un instrumento de gestión organizacional que una simple colección de datos estadísticos usados únicamente para efectos comunicativos o de rendición.

Frente al grado de aporte que las diferentes metodologías y aproximaciones de modelos de evaluación social analizados hicieron al diseño del CMI, se resalta que es la perspectiva social la más nutrida. La metodología SROI y el enfoque de capacidades (Desarrollo Humano) proveen la mayor parte de indicadores necesarios para analizar el "impacto social" de las intervenciones o proyectos. Estos dos enfoques, contribuyen claramente a evaluar aquellos objetivos estratégicos relacionados a la "mejora de calidad de vida" de las personas y las transformaciones sociales en periodos de tiempo más prolongados, relacionadas básicamente a la evolución de dimensiones como el nivel educativo, la salud, la vivienda, el trabajo y la autonomía. 
Por otro lado, los indicadores propuestos por la Guía G4 del Global Reporting Initiative (GRI), realizaron un significativo aporte a la perspectiva sostenibilidad financiera y a la perspectiva aprendizaje y crecimiento. Esta guía promueve enfáticamente la medición y comunicación de indicadores relacionados a la gestión eficiente y transparente de los recursos económicos, incluyendo las medidas relacionadas a las fuentes de financiamiento y captación de nuevos recursos. El GRI, también contribuye en la medición cuantitativa referente al bienestar, desarrollo y motivación de los colaboradores de la organización, siendo así, el origen de indicadores tan relevantes para el modelo relacionados a la formación del empleado y las tasas de rotación.

En cuanto a las propuestas de la auditoría social de REAS y del balance del bien común, ambas realizaron su máxima contribución a los indicadores del objetivo estratégico "Promoción de la responsabilidad social" incluido dentro de la perspectiva social. Estos dos balances fomentan mediciones relacionadas con la gestión responsable de proveedores, el adecuado comportamiento ecológico, la igualdad de género y la participación y transparencia en la información.

También es preciso anotar, que para la perspectiva procesos internos, ninguno de los enfoques de evaluación de desempeño social aportó indicadores que facilitaran el seguimiento a los respectivos objetivos estratégicos. Esto, se debe a que la cadena de valor de los procesos es muy única de cada entidad. Los objetivos asociados a esta perspectiva pueden variar significativamente según las líneas de trabajo de cada organización, de sus modelos de operación y de otros factores internos (capacidad, recursos, cultura organizacional, valores, etc.) o externos, (contexto económico, político o social en el que se desarrolla la intervención).

Dentro del análisis de la cadena de valor de la organización, y con el fin de definir los objetivos e indicadores a incluir dentro de la perspectiva de procesos internos, se vuelve entonces necesario identificar y estudiar aquellas variables, internas o externas, que de alguna manera pueden afectar el quehacer organizacional y, por tanto, sus actividades y procesos. En este sentido, para la definición de esta perspectiva dentro del CMI, fue crucial recurrir a indicadores propios de la organización (ya descritos en sus documentos de planificación) que permitieran controlar el logro de sus objetivos, de acuerdo sus particularidades.

Tanto las teorías de evaluación analizadas, como los modelos de Kaplan y Norton, otorgan una posición relevante a los stakeholders; en ningún caso, estos esquemas estudiados, contemplan "únicamente" los intereses del beneficiario y la organización, sino que también involucran las expectativas y demandas de otros grupos como donantes, comunidad y gobierno. La herramienta propuesta, recoge este principio de involucramiento de stakeholders y, en sus cuatro perspectivas, considera los intereses de los mismos. En la perspectiva social, se refleja en mayor parte las expectativas de los beneficiarios, donantes y comunidad, mientras que, en las perspectivas de sostenibilidad financiera, procesos internos y aprendizaje y crecimiento, se contemplan intereses más propios de la organización y sus colaboradores. 
Si bien, durante la actividad de validación, se percibió en los testimonios de los gestores entrevistados una clara aceptación de los potenciales beneficios de la herramienta propuesta, también se admitió el reto que significaría la implementación de la misma, especialmente en su versión avanzada. Adoptar el CMI con la totalidad de sus indicadores, exige a las entidades desarrollar procesos de formación para sus equipos en cuanto las diversas técnicas de medición y métodos como la línea de base y las evaluaciones ex post.

En las entrevistas realizadas, también pudo ratificarse las debilidades generales que tienen las entidades sociales (gestores o técnicos) en cuanto a la comprensión de la cadena de resultados y, por tanto, no hay un pleno reconocimiento del impacto generado por los proyectos o intervenciones y su aporte a una "transformación social" en un largo plazo. Algunos de los indicadores de impacto relacionados a la calidad de vida, fueron cuestionados por los entrevistados. Estos, manifestaron que, aunque "sería ideal" lograr y demostrar mejoras en las capacidades personales y condiciones de vida de los públicos atendidos, no era posible asumir tal compromiso, dado el alcance de sus intervenciones y la cantidad de factores externos que condicionan dicho impacto.

\section{Conclusiones y recomendaciones}

Aunque los objetivos iniciales de este trabajo se orientaban al diseño de un modelo estándar de Mapa Estratégico y CMI, las validaciones realizadas sugieren que no es posible "generalizar" completamente el modelo, aun en organizaciones que tienen una misión casi idéntica (fomento del empleo inclusivo). A pesar de que se evidencia gran adaptabilidad dentro de las perspectivas social, de sostenibilidad financiera y de aprendizaje y crecimiento, no es posible adaptar los objetivos e indicadores de la perspectiva procesos internos en su totalidad. Estos procesos y las estrategias que se establecen en dicha perspectiva varían de una organización a otra, ya que se encuentran ligados al quehacer propio de cada entidad y a su modelo de operación particular.

Por lo anteriormente expuesto se recomienda que antes de la implementación del modelo, se realice una revisión y, si es del caso, una adecuación del mapa y sus objetivos estratégicos, igual que las valoraciones y ponderaciones de los indicadores, ya que estas, también pueden variar en función de las necesidades y prioridades de cada organización. El instrumento de planificación y seguimiento desarrollado es lo suficientemente explicativo y flexible para que los gestores puedan adaptarlo según sus expectativas, recursos y capacidades. 
Los modelos de evaluación social analizados contribuyen de forma "parcial" a la construcción del $\mathrm{CMI}$. Aunque en conjunto, estos esquemas proponen cientos de indicadores, muchos de ellos, no se relacionan de manera directa con los objetivos estratégicos de las organizaciones analizadas. El CMI diseñado recoge de todos estos esquemas, aquellos indicadores relevantes, que además de cumplir con una función informativa para los grupos de interés, permiten realizar seguimiento y control al cumplimento de los objetivos estratégicos, la misión y la visión de la organización.

La propuesta de adaptación de conceptos del Cuadro de Mando Integral (CMI) al sector social, producto de esta investigación, es comprendida y aceptada por las organizaciones en las cuales se realiza la validación. La percepción de los gestores es positiva. Estos manifiestan que el CMI planteado podría, además de subsanar muchas de sus debilidades de corte administrativo, aportar a los procesos de comunicación interna, externa y a la toma de decisiones para el beneficio final de los colectivos atendidos y la satisfacción de otros grupos de interés.

A pesar de la generalizada aceptación del modelo, el panorama que se vislumbra para la implementación de este tipo de herramientas es complejo. La limitación de recursos y capacidades internas de las organizaciones, representan el mayor obstáculo para internalizar una cultura de seguimiento y control que optimice la toma de decisiones. El "caos organizado", tal y como lo manifestó una de las entidades, parece ser el común denominador de las formas de gestión, esto es, que existe tanto afán y compromiso por las tareas críticas (ejecución de las intervenciones directamente con los beneficiarios), que pocos recursos de tiempo y personal se dedican a funciones de corte más estratégico y de mejoramiento continuo.

Adicionalmente, el débil conocimiento en técnicas de medición de impacto social y la insuficiente concientización sobre el aporte que los proyectos y actividades de la entidad, realizan a la transformación social de una comunidad, hace que los instrumentos de medición sean cortoplacistas. No existe una clara diferenciación entre aquellos indicadores de "resultado" y de "impacto" y, por tanto, estos últimos son un poco subestimados a la hora de considerarlos en la planificación estratégica. Así pues, los gestores deberán reconocer el reto, tomar conciencia, y a la vez, asumir las condiciones y requisitos necesarios para asegurar que la evaluación sea una herramienta útil en la toma de decisiones orientadas a la maximización del beneficio social. Estas condiciones se describen a continuación:

En primer lugar, se debe asegurar que la evaluación sea vista como un proceso constante y de largo plazo, ya que los tiempos necesarios para poder comprobar los efectos de las intervenciones y los cambios que se producen en los individuos, las comunidades y poblaciones atendidas (impacto), por lo general, superan los dos o tres años después de finalizada la intervención. Este tiempo puede ser incluso superior, según el tipo de proyecto o acción social que desarrolle la organización. 
En segundo lugar, es pertinente incluir dentro de las mediciones, la valoración de los activos intangibles (involucrando indicadores y técnicas cualitativas). Por medio de la ejecución de la estrategia, la entidad debe estar aportando al incremento de estas capacidades, tanto en las comunidades atendidas, como en el grupo de colaboradores. Se vuelve imprescindible entonces, realizar seguimiento a los objetivos estratégicos asociados a estos intangibles, ya que se constituyen en los mayores generadores de valor a largo plazo y los que más podrían contribuir a la transformación social.

En tercer y último lugar, debe considerarse, que tanto la implementación del CMI, como cualquier otro proceso de mejoramiento interno que se pretenda llevar a cabo en organizaciones de este tipo, deberá ser primero dimensionado según el escenario especifico de cada entidad y planificado de una manera progresiva, a fin de evitar el rechazo por parte de los miembros de las organizaciones y garantizar que se materialicen los beneficios del modelo o instrumento que se adopte.

A partir de los resultados obtenidos en este trabajo, y de acuerdo con la experiencia desarrollada en las organizaciones involucradas, se proponen algunos temas o líneas de investigación para futuros estudios. El primero, es la profundización en teorías y modelos de evaluación de impacto social, que puedan aportar herramientas a este tipo de entidades y permitan entender los procesos de cambio social y la contribución que hacen las intervenciones al desarrollo sostenible de las comunidades y localidades.

En esta misma línea sería interesante explorar, dentro de los métodos de planificación y evaluación llevados a cabo por las entidades sociales, como se realiza el alineamiento con los actuales Objetivos de Desarrollo Sostenible (ODS) y analizar, además, si los indicadores vinculados a estos ODS podrían aportar al Cuadro de Mando Integral.

Finalmente, siendo conscientes de que el trabajo desarrollado no se extendió a un alto número de organizaciones, se podría ampliar el alcance en una futura investigación, validando el CMI con otras entidades e incluyendo las voces de otros grupos de interés (Stakeholders). Esto, podría dar lugar reflexiones más profundas y realizar propuestas de herramientas cada vez más integrales, orientadas a la maximización del impacto social de las organizaciones del tercer sector. 


\section{Referencias bibliográficas}

AUSTIN, J., GUTIERREZ, R., OGLIASTRI, E. \& REFICCO, E. (2006): Gestión efectiva de emprendimientos sociales: lecciones extraídas de empresas y organizaciones de la sociedad civil en Iberoamérica, Washington, DC: Banco Interamericano de Desarrollo.

BOSMA, N., SCHOTT, T., TERJESEN, S. \& KEW, P. (2016): Special Topic Report: Social Entrepreneurship. Global Entrepreneurship Monitor. Recuperado de https://www.gemconsortium. org/report/49542

BLACHFELLNER, M., DROSG-PLÖCKINGER, A., FIEBER, S., HOFIELEN, G., KNAKRÜGGE, L., KOFRANEK, M., ... TERIETE, M. (2017): Manual del Balance del Bien Común 5.0. Recuperado de https://economiadelbiencomun.org

COLINA, J. \& SENIOR, A. (2008): "Balance social. Instrumento de análisis para la gestión empresarial responsable", Multiciencias, 8 (Extraordinario), 71-77.

CURTO, M. (2012): "El emprendimiento social: Estructura organizativa, retos y perspectivas de futuro", Cuadernos de la Cátedra "La Caixa» de Responsabilidad Social de la empresa y Gobierno Corporativo, 14, 1-24.

DÍAZ, M., MARCUELLO, C. \& MARCUELLO, Ch. (2012): "Empresas sociales y evaluación del impacto social", CIRIEC-España, Revista de Economía Pública, Social y Cooperativa, 75, 179-198.

DRUCKER, P.F. (1986): Management: Tasks, Responsibilities, Practices. New York: Truman Talley Books.

DRUCKER, P.F. (2012): The practice of management. New York: Routledge.

FELBER, C. (2012): La economía del bien común, Barcelona: Deusto.

GARRIGA, E. (2013): Impacto Social: Un modelo en base a capacidades. Recuperado de https:/l www.fundacionseres.org/DocGrupoTrabajoPub/garrigadocumentofinal.pdf

GERTLER, P.J., MARTíNEZ, S., PREMAND, P., RAWLINGS, L.B. \& VERMEERSCH, C.M. (2017): La evaluación de impacto en la práctica, 2. Ed. Washington, DC: Banco Interamericano de Desarrollo y Banco Mundial. DOI:10.1596/978-1-4648-0888-3.

GLOBAL REPORTING INITIATIVE (2013): Introducción a la G4. La nueva generación de memorias de sostenibilidad. Recuperado de: https://www2.globalreporting.org/standards/g4/Pages/default. aspx 
GLOBAL REPORTING INITIATIVE (2018): "GRI Standards". Recuperado el 15 de diciembre de 2018 de https://www.globalreporting.org/standards/.

HILL, C.W. \& JONES, G.R. (2010): Strategic management: an integrated approach, 9th ed., Delhi: CENGAGE Learning.

JAHANTIGH, F.F., MALMIR, B. \& AVILAQ, B.A. (2018): "An integrated approach for prioritizing the strategic objectives of balanced scorecard under uncertainty", Neural Computing and Applications, 29(6), 227-236. DOI: 10.1007/s00521-016-2509-z.

KAPLAN, R. \& NORTON, D. (1996): El cuadro de Mando Integral. The Balanced Scorecard, 2. ed., Barcelona: Gestión 2000.

KAPLAN, R. \& NORTON, D. (2004): Mapas Estratégicos: convirtiendo los activos intangibles en resultados tangibles, Barcelona: Ediciones Gestión 2000.

LEADBEATER, C. (1997): The rise of the social entrepreneur, London: Demos.

MÉNDEZ, J.C., AYALA, H.J. \& PALACIOS, T.Y. (2019): "El Balanced Scorecard en el Desarrollo de los Negocios Familiares", INNOVA Research Journal, 4(33), 94-107. DOI: 10.33890/innova. v4.n3.2019.963.

MESSEGHEM, K., BAKKALI, C., SAMMUT, S. \& SWALHI, A. (2018): "Measuring Nonprofit Incubator Performance: Toward an Adapted Balanced Scorecard Approach", Journal of Small Business Management, 56(4), 658-680. DOI: 10.1111/jsbm.12317.

MORENO, A. (2012): Manual básico sobre el balance del bien común. Recuperado de https://tinyurl. com/yyayeghq.

MURILLO, L.M. (2016): Diseño de un Cuadro de Mando Integral para la Gestión del Impacto Social de Entidades y Proyectos Sociales Orientados al Incremento del Nivel de Empleabilidad de Colectivos en Riesgo de Exclusión. Universidad Politécnica de Valencia. Recuperado de https:// riunet.upv.es/handle/10251/67786.

NARRILLOS, H. (2010): "EI SROI (social return on investment): Un método para medir el impacto social de las inversiones", Análisis financiero, 113, 34-43.

NICHOLLS, J., LAWLOR, E., NEITZER, E. \& GOODSPEED, T. (2012): A guide to Social Return on Investment. Recuperado de http:/www.socialvalueuk.org/resources/sroi-guide/.

OECD (2002): Evaluation and Aid Effectiveness No. 6 - Glossary of Key Terms in Evaluation and Results Based Management. Paris: OCDE Publishing. DOI:10.1787/9789264034921-en-fr.

ORTIZ, A. \& RIVERO, G. (2007): "Desmitificando la Teoría de Cambio", Building Capacity Worldwide Pact.

PNUD (1990): Desarrollo Humano, Informe 1990. Bogotá: Tercer Mundo Editores. 
PNUD (2018): Índices e indicadores de desarrollo humano. Actualización estadística de 2018. Recuperado de http://hdr.undp.org/sites/default/files/2018_human_development_statistical_ update_es.pdf

RED DE REDES DE ECONOMÍAALTERNATIVAY SOLIDARIA(2018): "Auditoría Social". Recuperado el 16 de septiembre de 2018 de http://www.auditoriasocial.net/

RETALOZA, I. (2010): Teoría de Cambio. Un enfoque de pensamiento-acción para navegar en la complejidad de procesos de cambio social. Recuperado de http://www.dhl.hegoa.ehu.es/ recursos $/ 545$

RODRÍGUEZ DÍAZ, F.J. \& REY GARCÍA, M. (2017): "La evaluación de desempeño en las fundaciones: retos y herramientas", Boletín de Estudios Económicos, LXXII(220), 39-164.

SÁNCHEZ, F. (2003): "Planificación estratégica y gestión pública por objetivos", SERIE gestión pública Instituto Latinoamericano y del Caribe de Planificación Económica y Social - ILPES-, 32, 1-80.

SANTOS, M. \& FIDALGO, E. (2004): "Un análisis de la flexibilidad del cuadro de mando integral (CMI) en su naturaleza de las organizaciones", Revista Iberoamericana de Contabilidad de Gestión, 2(4), 1-21.

SEN, A. (2000): Desarrollo y Libertad. Planeta.

SPEAR, R. (2001): "El balance social en la economía social . Enfoques y problemática", CIRIECEspaña, Revista de Economía Pública, Social y Cooperativa, 39, 9-24. 


\section{Apéndice A. Tabla A1. Listado completo de indicadores del CMI por perspectiva y objetivo estratégico}

\begin{tabular}{|c|c|c|c|c|}
\hline Objetivo / Indicador & Definición & Dimensión & $\begin{array}{c}\text { Puntaje } \\
\text { valoración }\end{array}$ & Nivel \\
\hline \multicolumn{5}{|c|}{ Perspectiva Social } \\
\hline \multicolumn{5}{|c|}{ OE: Mejora de la calidad de vida y reducción de la exclusión social } \\
\hline \multirow[t]{2}{*}{ Nivel de satisfacción } & $\begin{array}{l}\text { Porcentaje de beneficiarios(as) que se consideran } \\
\text { "Satisfechos" o "Muy satisfechos" con los servicios recibidos, } \\
\text { la intervención o proyecto. }\end{array}$ & Cuantitativo & 4,3 & $\|$ \\
\hline & $\begin{array}{l}\text { Percepción de los beneficiarios en cuanto a la satisfacción de } \\
\text { sus necesidades, resolución de problemas y expectativas. }\end{array}$ & Cualitativo & 4,3 & $\|$ \\
\hline $\begin{array}{l}\text { Variación de la renta } \\
\text { familiar }\end{array}$ & $\begin{array}{l}\text { Porcentaje de beneficiarios(as) que incrementan el valor de } \\
\text { su renta familiar en los últimos dos años. }\end{array}$ & Cuantitativo & 3,9 & III \\
\hline \multirow{2}{*}{$\begin{array}{l}\text { Calidad de la } \\
\text { vivienda }\end{array}$} & $\begin{array}{l}\text { Porcentaje de beneficiarios(as) que adquieren vivienda o } \\
\text { mejoran la calidad de la misma en los últimos dos años. }\end{array}$ & Cuantitativo & 3,9 & III \\
\hline & $\begin{array}{l}\text { Percepción de los(a) beneficiarios en cuanto a la mejora de la } \\
\text { calidad de su vivienda y la contribución del servicio o proyecto } \\
\text { en este aspecto. }\end{array}$ & Cualitativo & 3,9 & III \\
\hline \multirow[b]{2}{*}{ Calidad de la salud } & $\begin{array}{l}\text { Porcentaje de beneficiarios(as) que mejoran la calidad de su } \\
\text { salud en los últimos dos años. }\end{array}$ & Cuantitativo & 3,9 & III \\
\hline & $\begin{array}{l}\text { Percepción de los(a) beneficiarios en cuanto a la mejora de } \\
\text { la calidad de su salud y la contribución del servicio o proyecto } \\
\text { en este aspecto. }\end{array}$ & Cualitativo & 3,9 & III \\
\hline \multirow{2}{*}{$\begin{array}{l}\text { Incremento de la } \\
\text { autonomía }\end{array}$} & $\begin{array}{l}\text { Porcentaje de beneficiarios(as) que mejoran la autonomía } \\
\text { en los últimos dos años, entendida esta como la capacidad } \\
\text { del individuo (física, mental e intelectual) para desarrollar las } \\
\text { actividades básicas de la vida diaria (ABVD). }\end{array}$ & Cuantitativo & 3,9 & III \\
\hline & $\begin{array}{l}\text { Percepción de los(a) beneficiarios en cuanto al incremento de } \\
\text { su autonomía para el desarrollo de las actividades básicas de } \\
\text { la vida diaria (ABVD) }\end{array}$ & Cualitativo & 3,9 & III \\
\hline \multicolumn{5}{|c|}{ OE: Mejora nivel de empleabilidad } \\
\hline Nivel educativo & $\begin{array}{l}\text { Porcentaje de beneficiarios(as) que se alfabetizan y/o } \\
\text { incrementan su nivel educativo en los últimos dos años. }\end{array}$ & Cuantitativo & 3,9 & $\|$ \\
\hline \multirow{2}{*}{$\begin{array}{l}\text { Cualificación } \\
\text { profesional / laboral }\end{array}$} & $\begin{array}{l}\text { Porcentaje de beneficiarios(as) que incrementan su nivel de } \\
\text { competencias laborales en el último año. }\end{array}$ & Cuantitativo & 3,9 & $\|$ \\
\hline & $\begin{array}{l}\text { Percepción de los(a) beneficiarios en cuanto al incremento } \\
\text { de sus competencias laborales y la contribución del servicio o } \\
\text { proyecto en este aspecto. }\end{array}$ & Cualitativo & 3,9 & $\|$ \\
\hline \multicolumn{5}{|c|}{ OE: Incremento nivel de inserción laboral } \\
\hline Inserción laboral & $\begin{array}{l}\text { Número de los(a) beneficiarios(as) que se han integrado } \\
\text { laboralmente en el último año }\end{array}$ & Cuantitativo & 4,8 & 1 \\
\hline $\begin{array}{l}\text { Atribución de la } \\
\text { inserción laboral }\end{array}$ & $\begin{array}{l}\text { Porcentaje de los(as) beneficiario(as) integrados laboralmente } \\
\text { que atribuyen al proyecto o a los servicios recibidos, el logro } \\
\text { de esta inserción. }\end{array}$ & Cuantitativo & 3,8 & III \\
\hline
\end{tabular}




\begin{tabular}{|c|c|c|c|c|}
\hline \multicolumn{5}{|c|}{ OE: Incremento del autoempleo y la creación de empresas } \\
\hline $\begin{array}{l}\text { Autoempleo y creación } \\
\text { de empresas }\end{array}$ & $\begin{array}{l}\text { Número de nuevos negocios/empresas emprendidas } \\
\text { npor los(as) beneficiario(as), que se encuentran en actual } \\
\text { operación y que generan como mínimo un empleo } \\
\text { remunerado. }\end{array}$ & Cuantitativo & 3,9 & I \\
\hline \multicolumn{5}{|c|}{ OE: Mejora de oportunidades y estructura del mercado (más inclusivo) } \\
\hline \multirow[t]{2}{*}{$\begin{array}{l}\text { Oportunidades en el } \\
\text { mercado laboral }\end{array}$} & $\begin{array}{l}\text { Efectividad de la prospección de empresas (ratio de } \\
\text { empresas visitadas que presentan alguna oferta en los seis } \\
\text { meses posteriores a la visita, respecto al total de empresas } \\
\text { visitadas). }\end{array}$ & Cuantitativo & 3,9 & II \\
\hline & $\begin{array}{l}\text { Nivel de ajuste de la formación ofertada a las necesidades del } \\
\text { mercado y los nuevos modelos productivos. }\end{array}$ & Cualitativo & 3,8 & II \\
\hline \multicolumn{5}{|c|}{ OE: Creación de un modelo de trabajo colaborativo } \\
\hline \multirow{2}{*}{$\begin{array}{l}\text { Cooperación y modelo } \\
\text { de trabajo colaborativo }\end{array}$} & $\begin{array}{l}\text { Número de convenios o acuerdos vivos con redes, } \\
\text { universidades, empresas, clústeres e iniciativas de } \\
\text { transformación social. }\end{array}$ & Cuantitativo & 4,3 & I \\
\hline & $\begin{array}{l}\text { Nivel de cooperación con otras entidades que proporcionan } \\
\text { productos/servicios similares. }\end{array}$ & Cualitativo & 4,0 & II \\
\hline \multicolumn{5}{|c|}{ OE: Promoción de la Responsabilidad Social Empresarial } \\
\hline \multirow{5}{*}{ Promoción de la RSE } & Valor social (ratio SROI). & Cuantitativo & 3,8 & III \\
\hline & $\begin{array}{l}\text { Porcentaje de puestos de trabajo de la organización ocupados } \\
\text { por mujeres (u otros colectivos en riesgo de exclusión). }\end{array}$ & Cuantitativo & 3,8 & I \\
\hline & $\begin{array}{l}\text { Promoción del comportamiento ecológico de las personas } \\
\text { empleadas y concepción ecológica de los productos o } \\
\text { servicios (incluyendo servicios financieros ético - ecológicos). }\end{array}$ & Cualitativo & 4,0 & I \\
\hline & $\begin{array}{l}\text { Porcentaje de gasto en compras de bienes y servicios a } \\
\text { entidades "socialmente responsables" (proveedores evaluados } \\
\text { en función de criterios ambientales, sociales y adecuadas } \\
\text { prácticas laborales). }\end{array}$ & Cuantitativo & 3,8 & I \\
\hline & $\begin{array}{l}\text { Nivel de participación de los grupos de interés involucrados en } \\
\text { la toma de decisiones de la organización. }\end{array}$ & Cualitativo & 4,0 & II \\
\hline \multicolumn{5}{|c|}{ Perspectiva Sostenibilidad Financiera } \\
\hline \multicolumn{5}{|c|}{ OE: Mejora fuentes de financiamiento } \\
\hline Ingresos & Valor total de ingresos. & Cuantitativo & 3,9 & I \\
\hline $\begin{array}{l}\text { Subvenciones y } \\
\text { donaciones }\end{array}$ & $\begin{array}{l}\text { Porcentaje de ingresos vía subvenciones públicas, donaciones } \\
\text { y concesiones respecto al total de ingresos. }\end{array}$ & Cuantitativo & 4,1 & I \\
\hline $\begin{array}{l}\text { "Venta" de productos } 0 \\
\text { servicios }\end{array}$ & $\begin{array}{l}\text { o Porcentaje de ingresos vía "facturación" por venta de bienes o } \\
\text { prestación de servicios (sin fines lucrativos). }\end{array}$ & Cuantitativo & 3,9 & I \\
\hline \multicolumn{5}{|c|}{ OE: Racionalización de gastos de funcionamiento y optimización de recursos } \\
\hline Gastos e inversiones & Valor total de gastos e inversiones. & Cuantitativo & 3,9 & $\mathrm{I}$ \\
\hline Inversión social & $\begin{array}{l}\text { Porcentaje del gasto orientado a la inversión social } \\
\text { (directamente relacionado a la ejecución de proyectos y } \\
\text { programas para los grupos beneficiarios) }\end{array}$ & Cuantitativo & 5,0 & I \\
\hline $\begin{array}{l}\text { Gastos de } \\
\text { funcionamiento }\end{array}$ & Valor total de los gastos de funcionamiento y estructura. & Cuantitativo & 3,9 & I \\
\hline
\end{tabular}




\section{OE: Gestión coordinada y planificada de los recursos}

\begin{tabular}{lllll}
\hline $\begin{array}{l}\text { Ejecución del } \\
\text { presupuesto de } \\
\text { ingresos }\end{array}$ & Porcentaje de ejecución de los ingresos. & 3,9 & Cuantitativo \\
\hline $\begin{array}{l}\text { Ejecución del } \\
\text { presupuesto de gastos }\end{array}$ & Porcentaje de ejecución de los gastos. & Cuantitativo & 3,9 & I \\
\hline
\end{tabular}

\section{Perspectiva Procesos Internos}

\section{OE: Mejora del conocimiento del mercado laboral}

\section{Conocimiento}

necesidades del

mercado laboral
Nivel actual del conocimiento sobre las necesidades del mercado laboral en la región de cobertura.
Cualitativo

4,2

II

\section{OE: Cobertura y calidad de los servicios}

\begin{tabular}{|c|c|c|c|c|}
\hline \multicolumn{2}{|c|}{ Personas beneficiarias Número de beneficiarios(as) de los servicios o proyectos. } & \multirow{2}{*}{$\begin{array}{l}\text { Cuantitativo } \\
\text { Cuantitativo }\end{array}$} & \multirow{2}{*}{$\begin{array}{l}5,0 \\
3,8\end{array}$} & \multirow[b]{2}{*}{$\|$} \\
\hline $\begin{array}{l}\text { Cobertura de los } \\
\text { servicios }\end{array}$ & $\begin{array}{l}\text { Porcentaje de cobertura de los servicios (en relación con la } \\
\text { demanda del servicio en la comunidad o región). }\end{array}$ & & & \\
\hline $\begin{array}{l}\text { Nivel de participación } \\
\text { y asistencia de los } \\
\text { beneficiarios }\end{array}$ & $\begin{array}{l}\text { Porcentaje de asistencia por parte de los beneficiarios } \\
\text { inscritos a las actividades programadas. }\end{array}$ & Cuantitativo & 4,3 & I \\
\hline Calidad del servicio & $\begin{array}{l}\text { Número de quejas presentadas por las personas beneficiarias } \\
\text { referentes a la prestación de los servicios. }\end{array}$ & Cuantitativo & 3,8 & I \\
\hline \multicolumn{5}{|c|}{ OE: Programas formativos de alto valor } \\
\hline $\begin{array}{l}\text { Nivel de acreditación } \\
\text { de la formación }\end{array}$ & $\begin{array}{l}\text { Porcentaje de participantes que obtienen acreditaciones } 0 \\
\text { certificados de profesionalidad. }\end{array}$ & Cuantitativo & 4,1 & II \\
\hline \multicolumn{5}{|c|}{ OE: Orientación e itinerarios de inserción personalizados } \\
\hline $\begin{array}{l}\text { Personalización del } \\
\text { servicio }\end{array}$ & $\begin{array}{l}\text { Porcentaje de beneficiarios con itinerario personalizado } \\
\text { abierto. }\end{array}$ & Cuantitativo & 4,4 & I \\
\hline \multicolumn{5}{|c|}{ OE: Fortalecimiento del emprendimiento } \\
\hline $\begin{array}{l}\text { Orientación al } \\
\text { emprendimiento }\end{array}$ & $\begin{array}{l}\text { Número de actividades ejecutadas orientadas al } \\
\text { fortalecimiento del emprendimiento. }\end{array}$ & Cuantitativo & 4,6 & I \\
\hline $\begin{array}{l}\text { Generación de ideas } \\
\text { y planes de nuevos } \\
\text { negocios }\end{array}$ & $\begin{array}{l}\text { Porcentaje de nuevas ideas/planes de negocio generados por } \\
\text { las personas beneficiarias de los servicios o proyectos. }\end{array}$ & Cuantitativo & 4,8 & I \\
\hline \multicolumn{5}{|c|}{ OE: Ejecución de proyectos especiales } \\
\hline \multirow{2}{*}{$\begin{array}{l}\text { Eficacia en la } \\
\text { ejecución de } \\
\text { "proyectos especiales" }\end{array}$} & $\begin{array}{l}\text { Porcentaje de avance en la ejecución de proyectos/ } \\
\text { programas. }\end{array}$ & Cuantitativo & 4,7 & I \\
\hline & $\begin{array}{l}\text { Nivel de avance del proyecto, incluyendo todos sus objetivos } \\
\text { y componentes. }\end{array}$ & Cualitativo & 4,7 & I \\
\hline
\end{tabular}

OE: Promoción y difusión de los programas

Efectividad de la

promoción y difusión Porcentaje de efectividad del plan de comunicaciones para la

de los servicios y promoción y difusión de los servicios y proyectos.

Cuantitativo

3,9

III

proyectos

\section{OE: Eficacia, eficiencia y mejora continua de los procesos}

\begin{tabular}{l}
$\begin{array}{l}\text { Aprovechamiento de } \\
\text { los recursos humanos }\end{array}$ \\
\hline
\end{tabular}




\begin{tabular}{|c|c|c|c|c|}
\hline $\begin{array}{l}\text { Aprovechamiento de } \\
\text { los recursos físicos }\end{array}$ & $\begin{array}{l}\text { Porcentaje de eficiencia de los recursos físicos } \\
\text { (aprovechamiento / ociosidad de los recursos). }\end{array}$ & Cuantitativo & 3,8 & ॥ \\
\hline \multicolumn{5}{|c|}{ Perspectiva Aprendizaje y Crecimiento } \\
\hline \multicolumn{5}{|c|}{ OE: Desarrollo del recurso humano } \\
\hline $\begin{array}{l}\text { Formación del recurso } \\
\text { humano }\end{array}$ & $\begin{array}{l}\text { Porcentaje de ejecución de los planes de "formación y } \\
\text { desarrollo" de los empleados de la organización. }\end{array}$ & Cuantitativo & 3,9 & $\|$ \\
\hline $\begin{array}{l}\text { Evaluación y mejora } \\
\text { del desempeño del } \\
\text { recurso humano }\end{array}$ & $\begin{array}{l}\text { Porcentaje de empleados evaluados y con planes de acción } \\
\text { establecidos para la mejora de su desempeño. }\end{array}$ & Cuantitativo & 3,9 & $\|$ \\
\hline \multirow{3}{*}{$\begin{array}{l}\text { Motivación y clima } \\
\text { laboral }\end{array}$} & $\begin{array}{l}\text { Porcentaje de empleados satisfechos con su labor y } \\
\text { motivados. }\end{array}$ & Cuantitativo & 4,2 & ॥ \\
\hline & Percepción del clima laboral. & Cualitativo & 4,2 & II \\
\hline & Porcentaje de rotación de empleados & Cuantitativo & 3,7 & 1 \\
\hline \multicolumn{5}{|c|}{ OE: Fortalecimiento de la comunicación interna } \\
\hline \multirow[t]{2}{*}{ Comunicación Interna } & $\begin{array}{l}\text { Porcentaje de empleados que perciben una comunicación } \\
\text { interna adecuada y eficaz. }\end{array}$ & Cuantitativo & 3,7 & $\|$ \\
\hline & Percepción sobre la comunicación interna & Cualitativo & 3,7 & II \\
\hline \multicolumn{5}{|c|}{ OE: Gestión del conocimiento } \\
\hline $\begin{array}{l}\text { Gestión del } \\
\text { conocimiento }\end{array}$ & $\begin{array}{l}\text { Grado de avance y relevancia de la gestión del conocimiento } \\
\text { en la organización (percepción colaboradores y otros grupos } \\
\text { de interés) }\end{array}$ & Cualitativo & 4,6 & III \\
\hline
\end{tabular}

Nota. OE: Objetivo estratégico.

FUENTE: Elaboración propia. 